\title{
Aerodynamic Comparison of Hyper-Elliptic Cambered Span (HECS) Wings with Conventional Configurations
}

\author{
Barry S. Lazos* \\ NASA Langley Research Center, Hampton, VA, 23681 \\ Kenneth D. Visser† \\ Clarkson University, Potsdam, NY, 13699
}

\begin{abstract}
An experimental study was conducted to examine the aerodynamic and flow field characteristics of hyper-elliptic cambered span (HECS) wings and compare results with more conventional configurations used for induced drag reduction. Previous preliminary studies, indicating improved L/D characteristics when compared to an elliptical planform prompted this more detailed experimental investigation. Balance data were acquired on a series of swept and un-swept HECS wings, a baseline elliptic planform, two winglet designs and a raked tip configuration. Seven-hole probe wake surveys were also conducted downstream of a number of the configurations. Wind tunnel results indicated aerodynamic performance levels of all but one of the HECS wings exceeded that of the other configurations. The flow field data surveys indicate the HECS configurations displaced the tip vortex farther outboard of the wing than the Baseline configuration. Minimum drag was observed on the raked tip configuration and it was noted that the winglet wake lacked the cohesive vortex structure present in the wakes of the other configurations.
\end{abstract}

\section{Nomenclature}

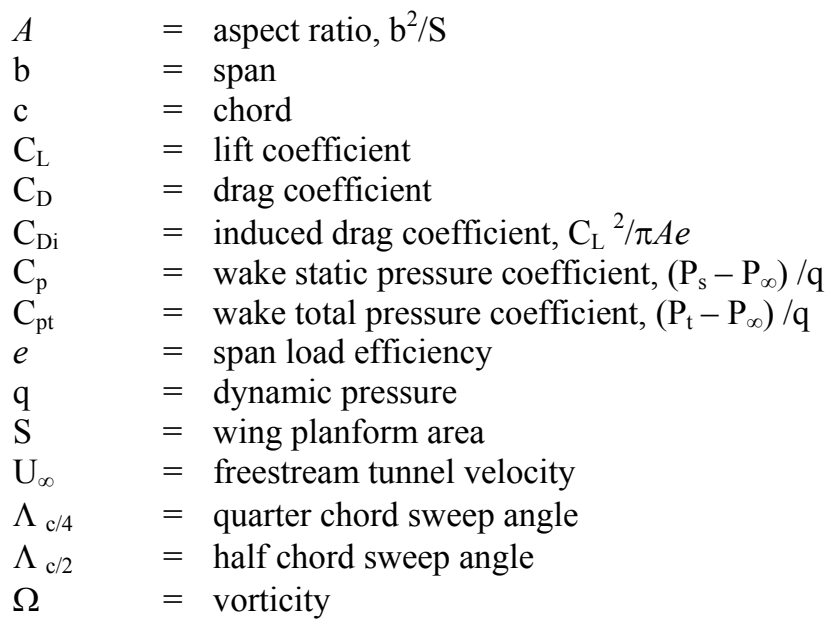

\section{Introduction and Motivation}

TMPROVEMENT in the aerodynamic performance for a given projected wing span has long been a goal of considerable interest. Two important inviscid calculations of performance were conducted by Munk ${ }^{1}$ and Cone. ${ }^{2}$ Munk determined that a planar wing of given span, with an efficiency defined as $e=\mathrm{C}_{\mathrm{L}}{ }^{2} / \pi A \mathrm{C}_{\mathrm{Di}}$, reached a maximal value of unity when the wing was elliptically loaded. Cone investigated numerous out-of-plane configurations and found some to result in efficiencies approaching 1.5. Many fixed geometry tip devices, including tailored wingtips, end plates, winglets, and tip sails have been investigated in an effort to reduce the induced drag and possibly

* Research Scientist, Flow Physics and Control Branch, AIAA Member.

$\dagger$ Associate Professor, Department of Mechanical and Aeronautical Engineering, Box 5725, AIAA Senior Member 
minimize the trailing vortex strength. Increasing the effective aspect ratio with devices such as endplates and winglets does help, although this is offset in part by the increase in viscous drag from the added wetted area. Welldesigned winglets ${ }^{3,4}$ can direct a component of their lift in the thrust direction, although the effect per se is to reduce the downwash, and tip sails have indicated the potential for reductions in induced drag. ${ }^{5}$ Jones $^{6}$ indicated that if the bending moment is constrained, winglets produce about as much drag savings as a planar tip extension. If, however, a winglet is optimized for minimum drag at a fixed span, it is equivalent to a planar wing with a span increase of about $45 \%$ of the winglet height. ${ }^{7}$ Much work has also been performed to study the effects of tailoring the wingtip planform geometry such as by shearing or raking the tip, ${ }^{8,9}$ or through the use of even more exotic geometry such as crescent shaped tips. ${ }^{10,11}$ Determining the drag savings for a given tip modification is not always an easy task, however. ${ }^{12}$ Numerous other investigations of wing performance have been performed using analytical, numerical, and experimental techniques many of which are mentioned in reviews by Henderson and Holmes ${ }^{13}$ and Rokhsaz. ${ }^{14}$

Recently, Lazos ${ }^{15}$ experimentally compared several biologically inspired wing configurations to a planar-elliptic Baseline wing. Several wing geometries were configured after a seagull in gliding flight, such as that shown in Fig. 1 , while taking into account the work of Cone. ${ }^{2}$ Lift-to-drag (L/D) measurements showed that one of the configurations, dubbed the HECS (Hyper-Elliptic Cambered Span) wing, provided a 4 percent improvement.

It is interesting to digress a moment to consider the possible reasons a seagull wing is shaped as it is. One can assume that the bird is somehow optimizing its energy consumption to provide the highest $\mathrm{L} / \mathrm{D}$ ratio with the minimum energy input. It would seem inefficient for the seagull to have to "force" its wing into maintaining a certain shape, as that would require more energy, so one could also assume this shape is a balance of the weight of the wing and the aerodynamic loading such that the best aerodynamics are achieved. If this is true, it implies that this type of geometry

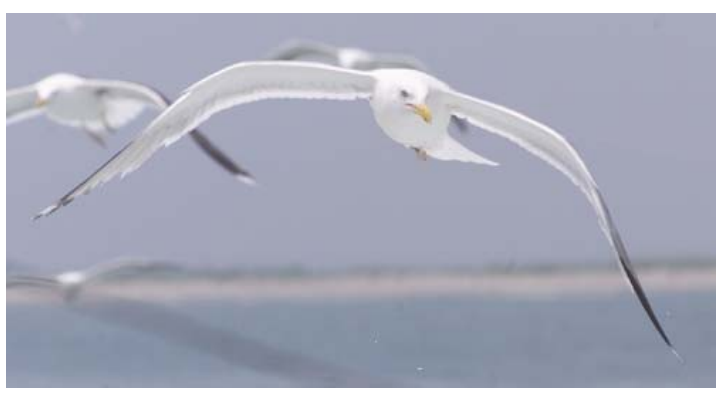

Figure 1. Seagull in gliding flight.

is "better" than would be had if the bird fully extended its wings to straight and level (or even upwards). It is the general human understanding, however, that if the bird did set its wings level, the wingspan would increase and, according to our understanding of induced drag, $\mathrm{C}_{\mathrm{Di}}$ would decrease and the $\mathrm{L} / \mathrm{D}$ ratio would increase. Instead, the seagull flies with a geometry that gives a span below what it has available! Does this imply that one might be able to reduce the drag, for a given lift, with a reduced effective span?

The previous results, and this line of thinking, has prompted the current, more detailed experimental investigation of the original HECS wing, along with several HECS wing variants, the original Baseline wing, two winglet configurations, and a raked tip wing in an effort to gain a better understanding of the flow physics of the effect of spanwise camber. Experimental force balance data were acquired on each wing and wake flow surveys were conducted with a seven-hole pressure probe on several of the configurations

\section{Experimental Models and Apparatus}

Experiments were conducted in the Basic Aerodynamics Research Tunnel (BART) at the NASA Langley Research Center. This facility is an open-circuit wind tunnel with a test section area of 28- $\mathrm{x} 40$-inches and a length of 120 inches. The facility was operated at a constant speed of $50 \mathrm{~m} / \mathrm{s}$ for all tests to yield a Reynolds number of 1 million per foot. Reference 16 provides detailed information on tunnel flow quality and indicates that turbulence levels are below 0.1 percent at near maximum free stream velocity.

\section{A. Model Definition and Construction}

Eight different configurations were tested. All models were attached to the same center body and all had a wing planform area of $112 \mathrm{in}^{2}$ with a wingspan of 28 inches. Hence each configuration had an aspect ratio of 7 . Here the wing reference area used to calculate the aspect ratio is the planform area of the wings alone $\left(112 \mathrm{in}^{2}\right)$ excluding any area projected into the center body. This definition of aspect ratio was chosen because it was expected to better represent the geometry differences between the wings. The airfoil section used was the SD7032, ${ }^{17}$ a low Reynolds number airfoil designed to mitigate separation problems through the use of a bubble ramp. The maximum wind tunnel blockage for all models was approximately 2.7 percent. 

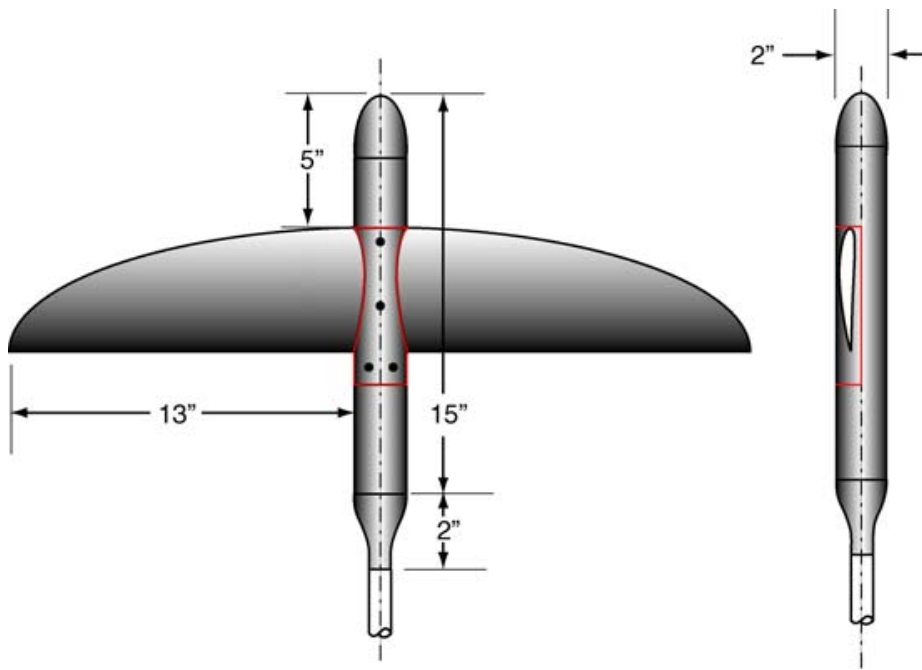

Figure 2. Baseline model geometry and center support.

Table 1. Model geometry specifications.

\begin{tabular}{lcc}
\hline \hline \multicolumn{1}{c}{ Wing model } & $\begin{array}{c}\text { Wing surface area, } \\
\mathbf{i n}^{2}\end{array}$ & MAC, in. \\
\hline Baseline & 114.21 & 4.67 \\
\hline HECSI & 117.91 & 4.45 \\
\hline HECSII & 117.91 & 4.45 \\
\hline HECSV & 126.69 & 4.46 \\
\hline HECSVI & 126.69 & 4.46 \\
\hline Raked Tip & 114.60 & 4.83 \\
\hline Winglet1 & 123.07 & 5.13 \\
\hline Winglet2 & 121.28 & 5.07 \\
\hline \hline
\end{tabular}

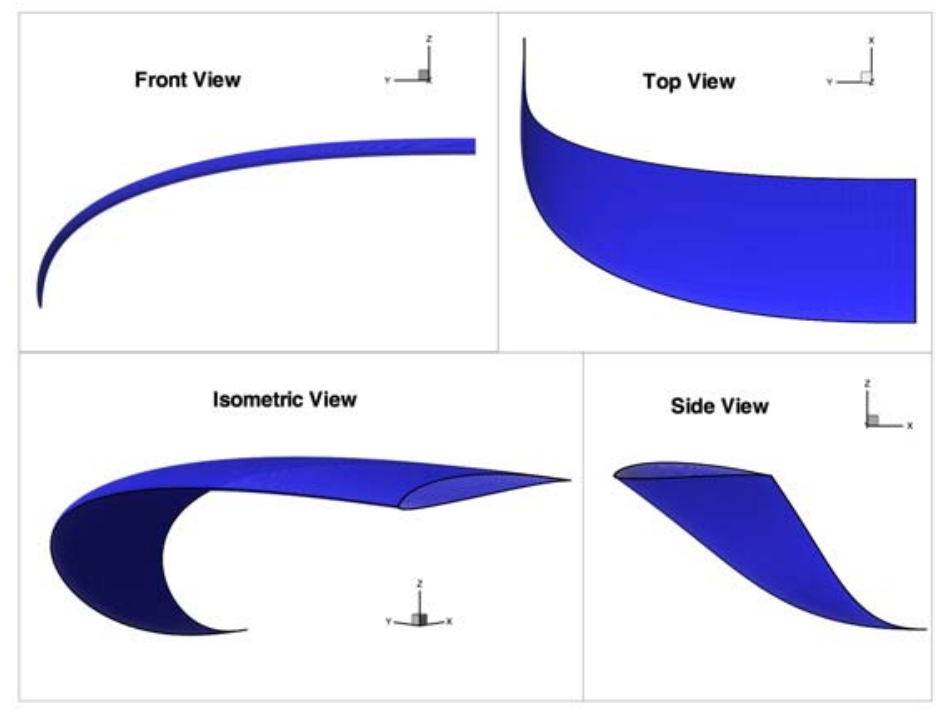

Figure 3. Multiple views of HECS1 configuration.
The Baseline configuration, illustrated in Fig. 2 attached to the centerbody, was a wing with an elliptic leading edge and a straight trailing edge. This configuration was chosen for the baseline since Smith ${ }^{18}$ demonstrated, through detailed computational studies, that this planform provided an induced drag efficiency factor closer to unity than any other planform tested with an elliptic chord distribution. Table 1 lists the wetted areas and mean-aerodynamic-chord for all the geometries and a brief description of the geometric details of each now follows.

\section{Hyper Elliptic Cambered Span Wings}

The HECS wing is a non-planar configuration with the curvature in the upstream view defined by a hyper-elliptic equation, that is, an elliptic equation with an exponent greater than two. The exact equation is given as:

$$
\left(\frac{z+4.727}{4.727}\right)^{2.5}+\left(\frac{y}{13}\right)^{2.5}=1
$$

where $z$ (in.) is in the upward normal direction and $y$ (in.) extends from root to tip. With the wing unfurled the leading-edge and trailingedge curvatures in the planview are defined, respectively, by the following hyper-elliptic equations:

Leading Edge:

$$
\left(\frac{9.552-x}{9.522}\right)^{2.5}+\left(\frac{y}{15.121}\right)^{2.5}=1
$$

Trailing Edge:

$$
\left(\frac{4.727-x}{4.727}\right)^{2.5}+\left(\frac{y}{15.121}\right)^{2.5}=1
$$

where $x$ (in.) is in the streamwise direction. The coordinate axis of the wing is located at the wing root leading edge. Figure 3 illustrates multiple views of the geometry. Since several modified HECS configurations were constructed for this test, the original, from Ref. 15, was renamed HECSI. The HECSII wing was identical to HECSI except that a gradual outboard twist was added to the tip. This feature was included to incline the lift vector of the tip toward the front in order to 
provide a thrust component. Twist began at about 10.3 inches along the span and continued to the wing tip. Curvature increments were defined by $\theta=5\left[(0.953)^{-\mathrm{n}}-1\right]$, where $\theta$ is in degrees and $1 \leq n \leq 34$. The HECSV wing varies from the HECSI in that it has a sweep defined by $\mathrm{X}$ transV $=\mathrm{X}$ transI+YtransI/tan(20), where Xtrans and Ytrans are the offsets in the $x$ and $y$ directions, respectively, of each of the 81 airfoil sections defining the wing. Sweep was added since Ref. 15 indicated the performance of a swept configuration, dubbed the shark wing, outperformed all other configurations. A planform image of the HECSV wing illustrating the sweep compared to HECSI (Fig. 3) is shown in Fig. 4. The HECSVI wing was a combination of the sweep in the HECSV wing and the tip twist in the HECSII wing.

\section{Winglet Models}

In order to approach the winglet design in a somewhat systematic measure, a series of design guidelines were culled from the literature and combined with the use of a vortex lattice code. The primary design guidelines used are listed below. These were used as the initial starting point for the designs.

- Winglet sweep set to $30^{\circ}$

- No twist along winglet (twist for lift at tip $=0$ )

- Small Cant angle (set to $15^{\circ}$ )

- $\quad$ Taper ratio $=0.6$

- Ratio of winglet chord to wingtip chord $=0.6$. It was suggested by Whitcomb ${ }^{3}$ that the leading edge of the winglet lie just behind the crest of main wing.

- Negative toe angle (toe out)

The geometries developed were also subject to several design constraints to make a "fair" comparison to the HECS wings including a planform area of $112 \mathrm{in}^{2}$, a semispan of the wing from root to tip of $13 \mathrm{in}$., and an aspect ratio of 7. It was also decided that the height in the $z$-direction would be set equal to the HECS wing of 4.727 inches to make for an equitable comparison. These parameters set most of the winglet geometry, except for the toe angle. Since the primary end goal of the design was to minimize the drag, both viscous and induced, for a given lift or lifts, it was decided to use a vortex lattice (VL) code to model the wing and vary the toe angle to give some guidance to the final design.

The VL code Tornado, from T. Melin ${ }^{19}$ at the Department of Aeronautics at the Royal Institute of Technology, in Stockholm, Sweden, is a fully three dimensional vortex lattice code with a flexible, free-stream following wake. Tornado allows a user to define multiple wings both cranked and twisted with multiple control surfaces. Each wing may have taper of both camber and chord. The Tornado solver solves for forces and moments, from which the aerodynamic coefficients are computed. Aerodynamic derivatives can be calculated with respect to: angle-of-attack, angle of sideslip, roll-, pitch-, and yaw-rotations, and control surface deflection.

The core method stems from Moran, ${ }^{20}$ but is modified according to Melin ${ }^{19}$ in order to accommodate a threedimensional solution and trailing edge control surfaces. The most notable change is the extension of the theory of the horseshoe vortex into the vortex-sling concept. The vortex sling is essentially a seven segment vortex line, which, for each panel, starts at infinity behind the aircraft, reaches the trailing edge, moves upstream to the hinge line of the trailing edge control surface, then forward to the quarter chord line of the panel in question, going across the panel and then back downstream in an analogous way. The issue of the wake passing through the geometry at certain flight conditions is resolved by a collocation point proximity detection routine which automatically removes the influence from a vortex thread passing too close to a collocation point.

The code allows a camber line to be defined based on a NACA 4 digit airfoil. The SD7032 has a maximum thickness-to-chord ratio $(\mathrm{t} / \mathrm{c})$ of 10.1 percent at an $x / \mathrm{c}=32.5$ percent. It has a maximum camber of 3.69 percent at $x / \mathrm{c}=41.91$ percent. The closest NACA 4 digit is a 4410 , which has a similar camber and $\mathrm{t} / \mathrm{c}$, although the maximum 
$\mathrm{t} / \mathrm{c}$ is at a chordwise location further downstream. Figure 5 illustrates the NACA 4410 along with the SD7032 for comparison. The NACA 4410 camber line was used for the VL design process.

A model was developed according to the above design guidelines and is illustrated in Fig. 6 . This initial winglet design had a simple taper of $\lambda=0.45$ with a quarter chord sweep, $\Lambda_{\mathrm{c} / 4}$, of $0^{\circ}$. The transition region between the winglet and the wing was set to 2 percent of the span. The winglet was set at the trailing edge and the leading edge was located at an $x / \mathrm{c}$ $=0.4$ of the wing tip chord, aft of the max $\mathrm{t} / \mathrm{c}$ by 8 percent of the tip chord and roughly at the point of maximum camber. Sweep of the winglet was $30^{\circ}$ with a taper of $\lambda=0.60$, and no twist or toe angle. An example of the resulting pressure distribution is shown in Fig. 6

The initial toe angle of $0^{\circ}$ was then varied to determine the minimum drag condition. Figure $7 \mathrm{a}$ illustrates the impact of toe angle. The results indicated that a toe angle of $-1.0^{\circ}$, denoted by the dotted line, was the optimum. The effect of twisting the outboard portion of the wing section next to the winglet and the wing itself was also investigated to determine if the design could be further refined. A linear twist of $1^{\circ}$ across the entire span was found to degrade the overall aerodynamic performance, but a small twist locally at the juncture of the wing and the winglet did improve the performance as shown in Fig. $7 \mathrm{~b}$ by the dotted line. The quarter chord sweep angle, $\Lambda_{\mathrm{c} / 4}, \mathrm{was}$

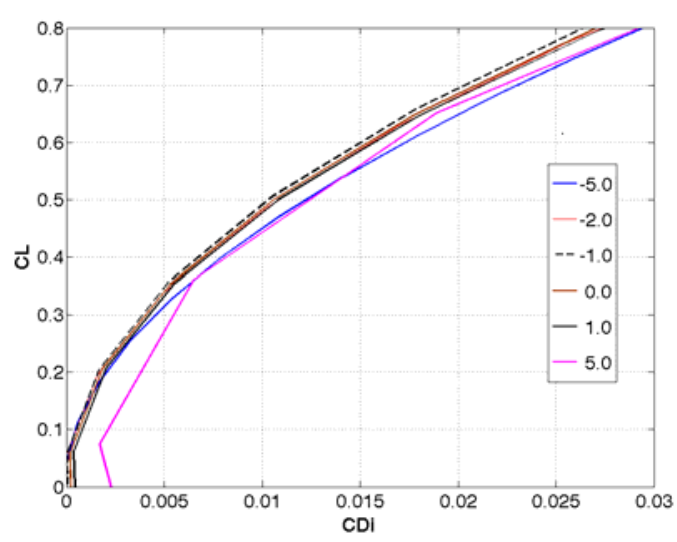

a) Effect of toe angle.

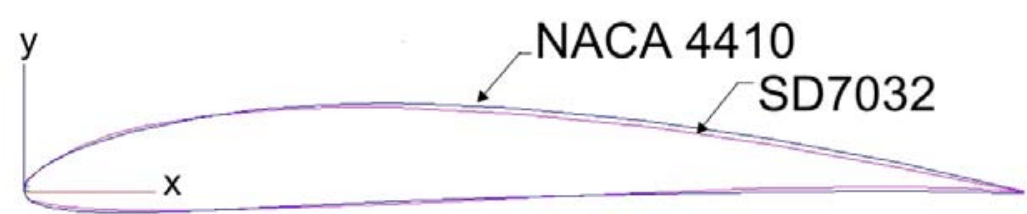

Figure 5. Comparison of SD7032 and NACA4410 airfoils.

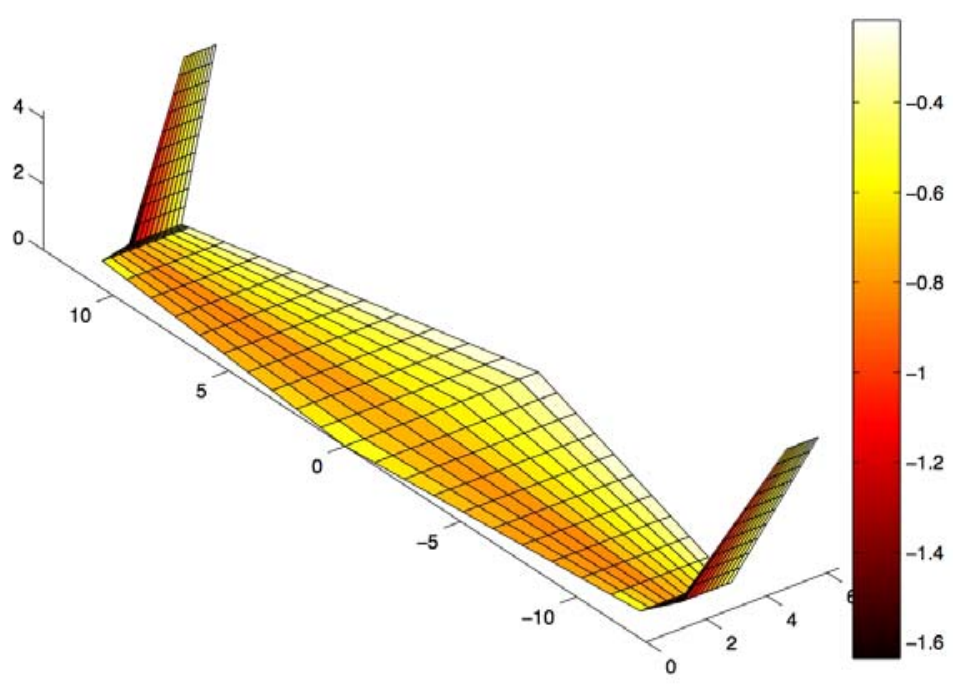

Figure 6. Cp distribution on initial winglet configuration, 2\% transition span length from wing to winglet, toe $=0^{\circ}, \alpha=3^{\circ}$.

Figure 7. Effect of toe and twist angle on initial winglet configuration. 
initially set to zero, however a small improvement was observed by "sweeping" the $\Lambda_{\mathrm{c} / 4}$ line to arrive at a $\Lambda_{\mathrm{c} / 2}=0^{\circ}$ and this was also incorporated into the final design. Complete details of the computations and the geometry can be found in Ref. 21.

The final lofts of the winglet geometries are illustrated in Fig. 8. Winglet2, shown in Fig. 8b, was designed with a smooth geometric transition from wing to winglet using the same design philosophy as above. This "blended winglet" is similar to the Boeing BBJ curved winglet and is also illustrated in Fig. 8b. Planform views of the geometries are shown in Fig. 9. Each design was constrained to a half span of 13 inches and a half area of $56 \mathrm{in}^{2}$. It

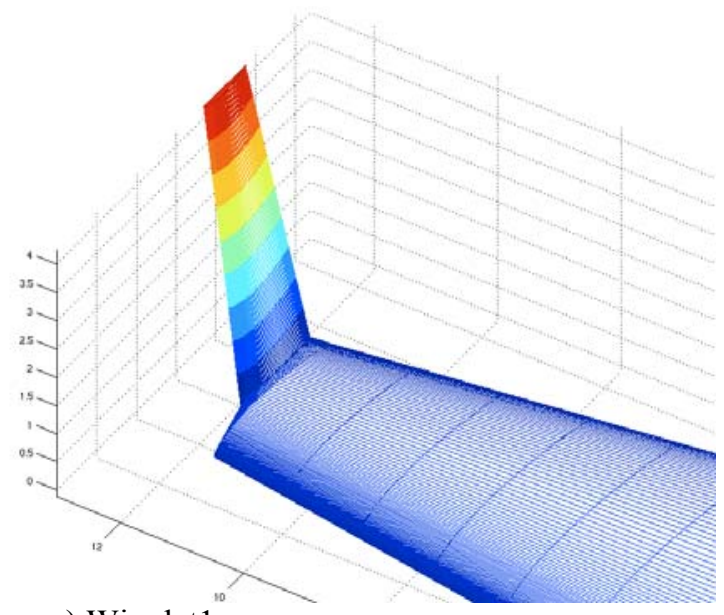

a) Winglet1

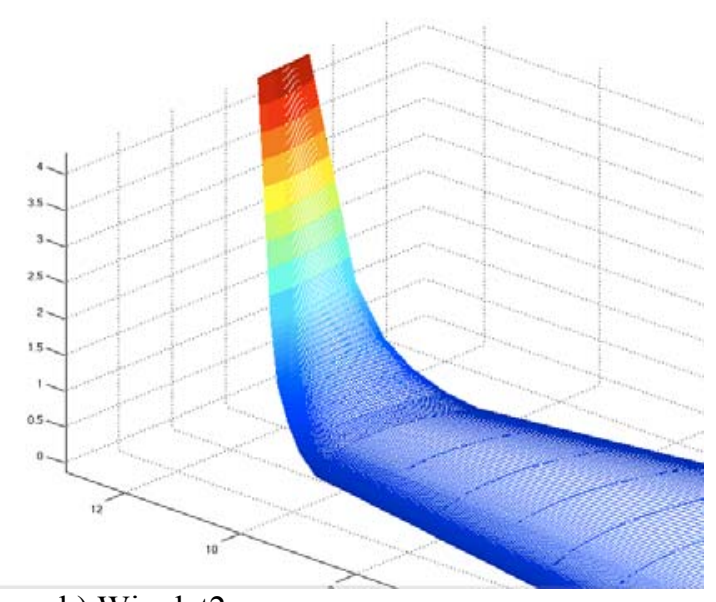

b) Winglet2

Figure 8. Final geometries of Winglet1 and Winglet2.

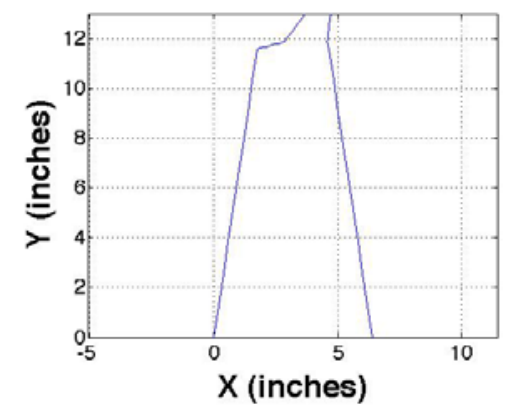

a) Winglet1

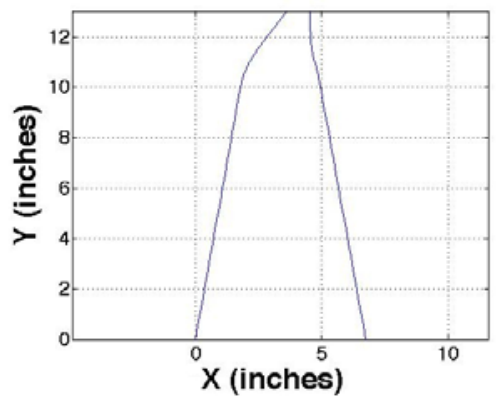

b) Winglet 2

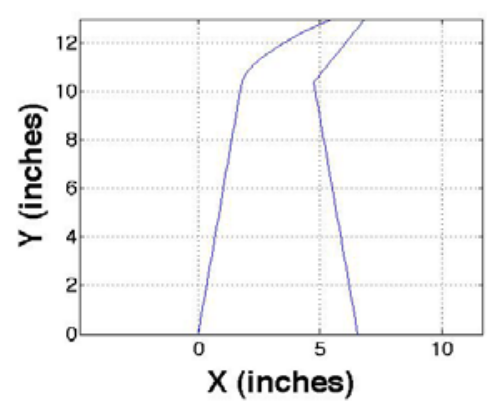

c) Raked Tip

Figure 9. Planform geometry of winglets and raked tip

should be noted here that the actual winglet height was 4.227 in. rather than 4.727 inches. The effects of this unfortunate design error will be discussed in a following section. A raked tip wing was also designed and constructed for comparison. A taper ratio of 0.45 was applied to the Raked Tip main wing, $0<y /(\mathrm{b} / 2)<0.9$, and to the tip, $0.9<y /(\mathrm{b} / 2)<1.0$, which comprised the outer $10 \%$ of the planform. A transition from $0^{\circ}$ to $10^{\circ}$ dihedral was incorporated into the tip. 


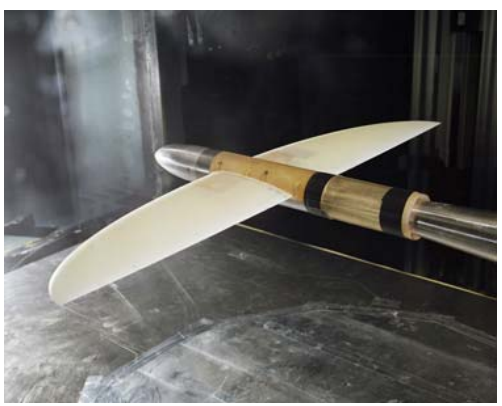

a) Baseline

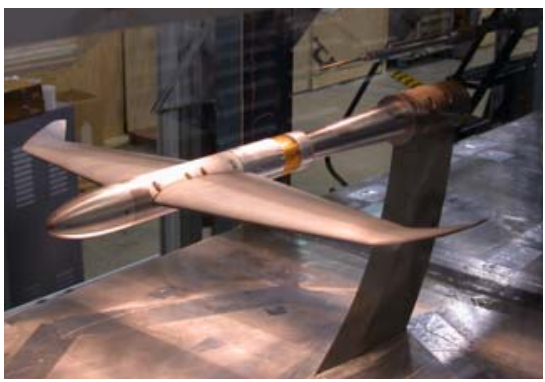

d) Raked Tip

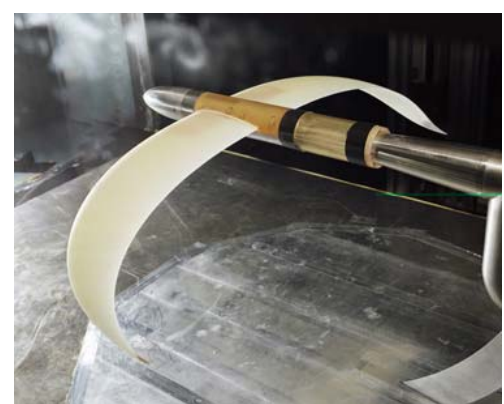

b) HECS1

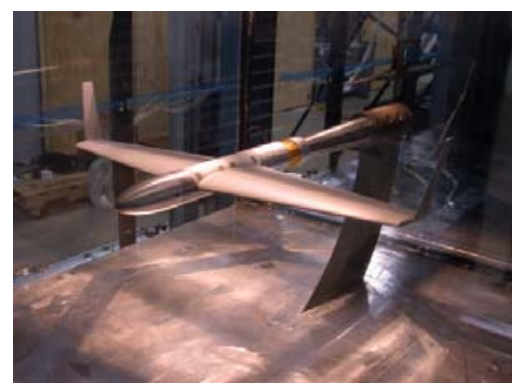

e) Winglet1

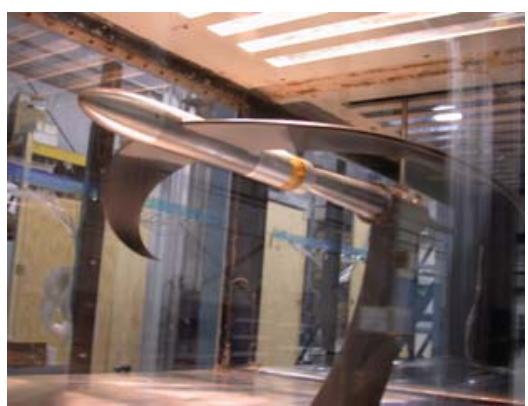

c) HECSV

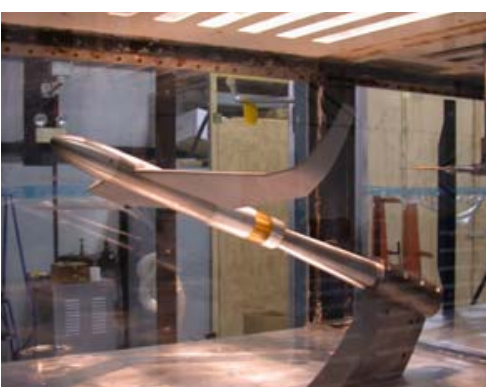

f) Winglet2

Figure 10. Sting mounted models in the BART tunnel.

\section{Model Construction}

Model wings were constructed in a three-step process to produce a durable configuration. First, each was formed in SL 5180 resin using stereo-lithography. The resin wings were then sanded and molded in a pliable polyurethane. The final wing configurations were then cast in a stiff polyurethane that has a flexural modulus of $432 \mathrm{ksi}$ and is more resistant to the adverse effects of humidity and ultraviolet light than SL 5180 resin. Models were constructed by attaching the left and right wings to a centerbody cap highlighted in red in Fig. 2. This allowed for screw attachment of each model to the same aluminum centerbody that was a generic cylindrical shape 2 inches in diameter with an elliptic nose cone. Figure 10 illustrates perspective views of six of the models sting-mounted in the tunnel test section.

\section{B. Wind Tunnel Data Acquisition and Reduction}

\section{Balance data}

Force and moment data were acquired using a six-component strain gauge balance. Before each run, weight tares were acquired by running the model through an angle-of-attack range under wind-off conditions. This provided an in situ measure of the balance electrical zeros, model static weight, and center-of-gravity location. Balance data were acquired for each model through an angle-of-attack range, $\alpha$, of $-4^{\circ}$ to $10^{\circ}$ in increments of $1^{\circ}$. This range was chosen to eliminate flutter and prevent balance over stressing. For each reading of the balance, data were acquired at a rate of 300 samples per second with 28 sets of 1000 samples acquired to provide a good mean measurement. Temperature and pressure data were acquired simultaneously with the balance data at the same sample rate to accurately calculate dynamic pressure at each angle-of-attack.

An accelerometer attached to the sting support was used to measure of the angle between the model and the horizontal. To account for deflections in the balance due to aerodynamic loads, deflection coefficients obtained from the balance calibration were used. Both normal force and pitching-moment coefficients were used in the correction and were given in units of degrees per pound and degrees per inch-pound, respectively.

Even with a precise measure of the angle that the model makes with the horizontal, an accurate measure of the angle-of-attack is not guaranteed since the flow in the test section is likely to be at some angle to the horizontal. In order to account for this accuracy error in the current study, the method of Barlow et al. ${ }^{22}$ was used. Each model was run several times through the angle-of-attack range in both an upright and inverted position. The mean lift 


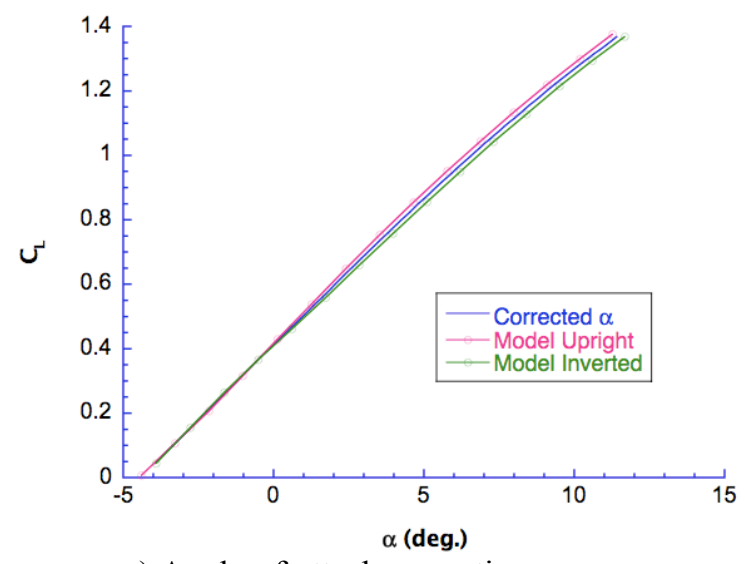

a) Angle-of-attack correction

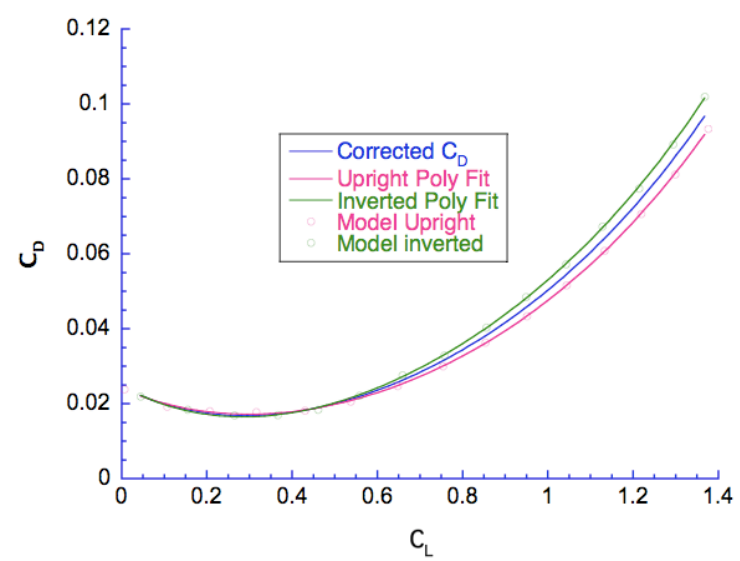

b) Drag coefficient correction

Figure 11. Correction strategy for aerodynamic loads.

coefficient was then plotted against the measured mean angle-of-attack for both the upright and inverted cases. The actual angle-of-attack was then determined at constant lift coefficient values by locating the mean between the upright and inverted cases. Drag coefficient values were corrected in a similar fashion by plotting lift coefficient versus mean drag coefficient for both the upright and inverted cases. A fourth-order polynomial was then fitted to the data, which provided a minor amount of smoothing. The actual drag coefficient was then determined as the mean between the polynomial curves running through the upright and inverted data. Figure 11 illustrates this correction strategy. For a more detailed explanation of this procedure see Lazos. ${ }^{15}$

\section{Wake Surveys}

Wake surveys were conducted with a seven-hole probe with a 0.125 in. diameter attached to the traverse system present at the BART. The traverse had five degrees of freedom with four degrees under computer control. It enabled movement of the probe with a translation resolution of $10 \mu \mathrm{m}$ and a yaw resolution of $0.01^{\circ}$. Due to the design of the models and centerbody the wing root leading edge for each model was at the same downstream location. Planes of wake data were acquired at a single downstream location corresponding to 20.375 inches downstream from this point. Course grids with 0.2 in. spacing were first acquired to ensure capture of the wake. Finer grids with $0.1 \mathrm{in}$.

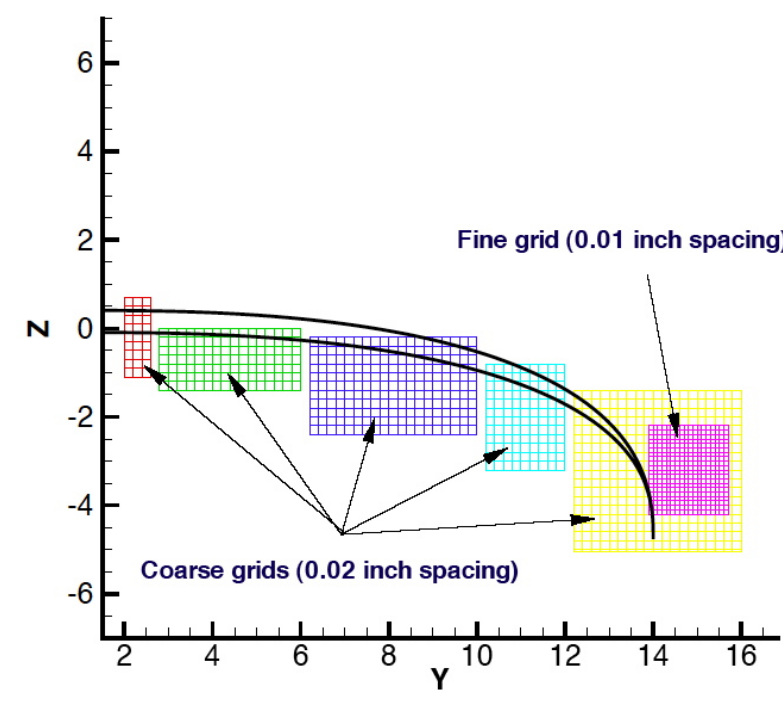

a) Original grids for HECSI wing.

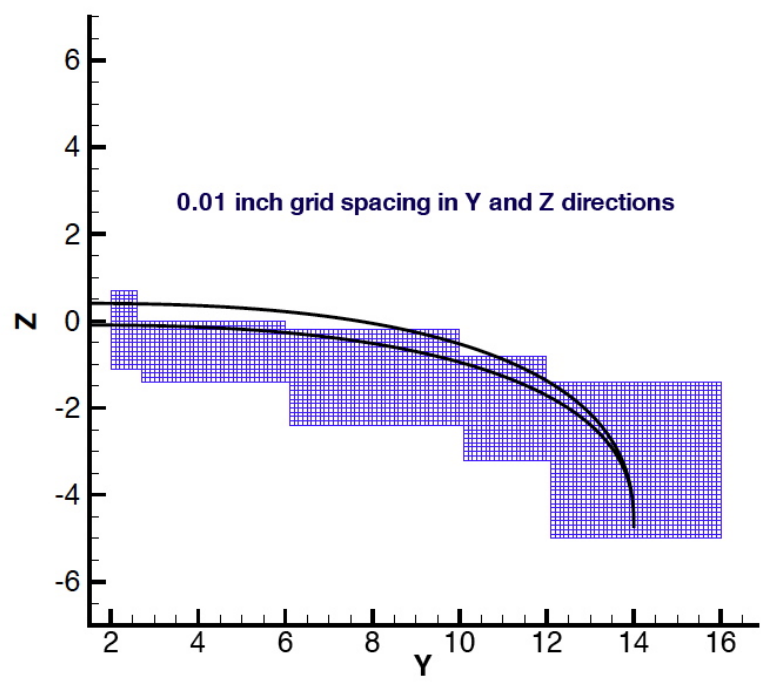

b) Re-sampled grid for HECSI wing.

Figure 12. Wind tunnel wake grid survey locations. 
spacing were then used to capture the detail of the shear layer and core regions. All models were set to the angle-ofattack corresponding to the maximum lift-to-drag for the upright model as indicated from the balance data. Since data were acquired for each configuration in several grids with two different resolutions, all data for a configuration were post processed into a single grid with a resolution of 0.1 inches using a weighted averaging technique. Examples of the original grids and the refined grids are shown for the HECSI wing in Fig. 12.

\section{Results and Discussion}

Force and moment data were acquired on the balance and wake data were acquired from a seven-hole probe. It should be noted here that balance data for all of the models repeated well along a smooth curve from run-to-run except for the HECSV data. For this configuration the data did not always follow a smooth curve, often times showing irregular behavior particularly in the drag bucket. For this study, only balance data that repeated well along a smooth curve are included in the averaged data set. There will be further discussion of this phenomenon as a variation in flow states is suspected. Wake data for the HECSV configuration is presented on the fine grid and the resampled coarse grid but not on the combined resampled grids. Data acquisition of the coarse and fine grid data was performed on two separate days and when the data were resampled together to the finer grid it was apparent

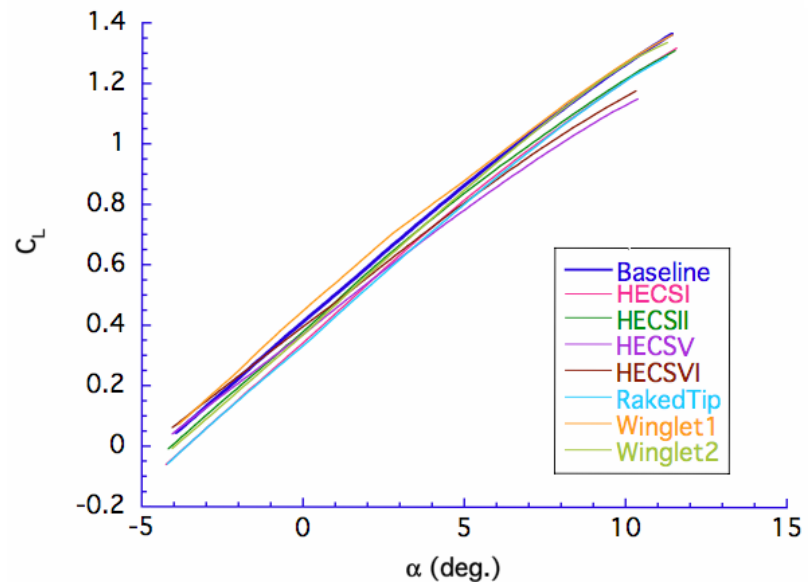

a) Full angle-of-attack range

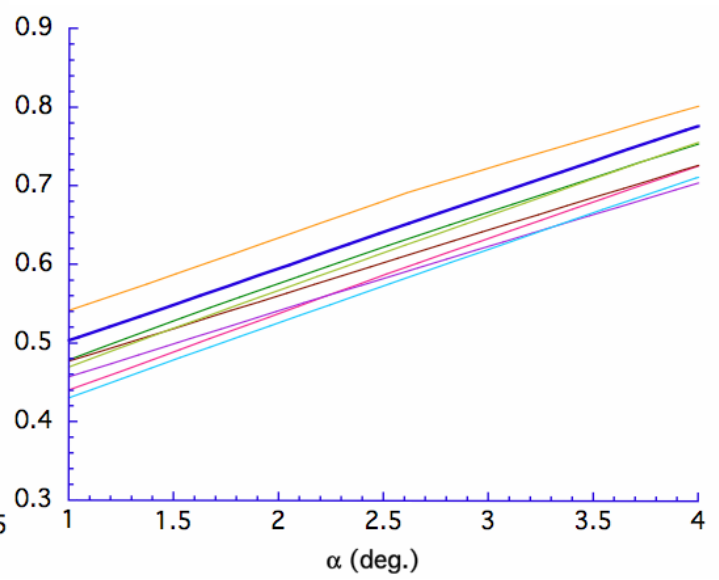

b) Angle-of-attack from $1^{\circ}$ to $4^{\circ}$

Figure 13. Wind tunnel lift behavior for each model configuration.

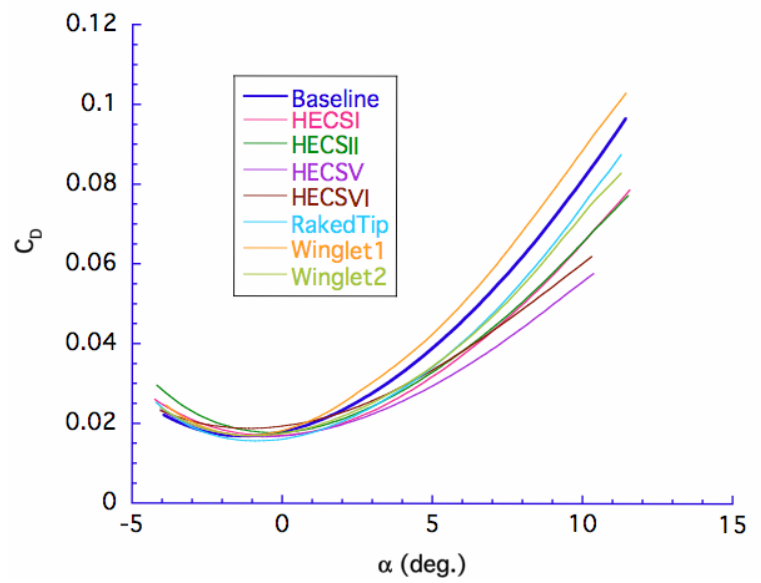

a) Full angle-of-attack range

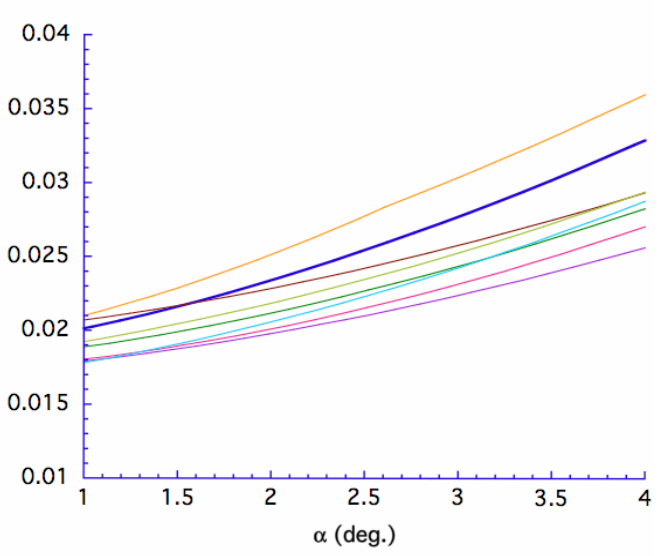

b) Angle-of-attack from $1^{\circ}$ to $4^{\circ}$

Figure 14. Wind tunnel drag behavior for each model configuration.

there were differences between the two data sets. This may be a result of changing flow states for this configuration, as stated above. Further study of this configuration is warranted since it provided the best lift-to-drag performance. 


\section{A. Aerodynamic Data}

The lift and drag behavior of each of the wings as a function of angle-of-attack was plotted in Figures 13 and 14 respectively. The lift plot in Fig. 13a shows the full range of data while Fig. 13b, on the right, highlights the region

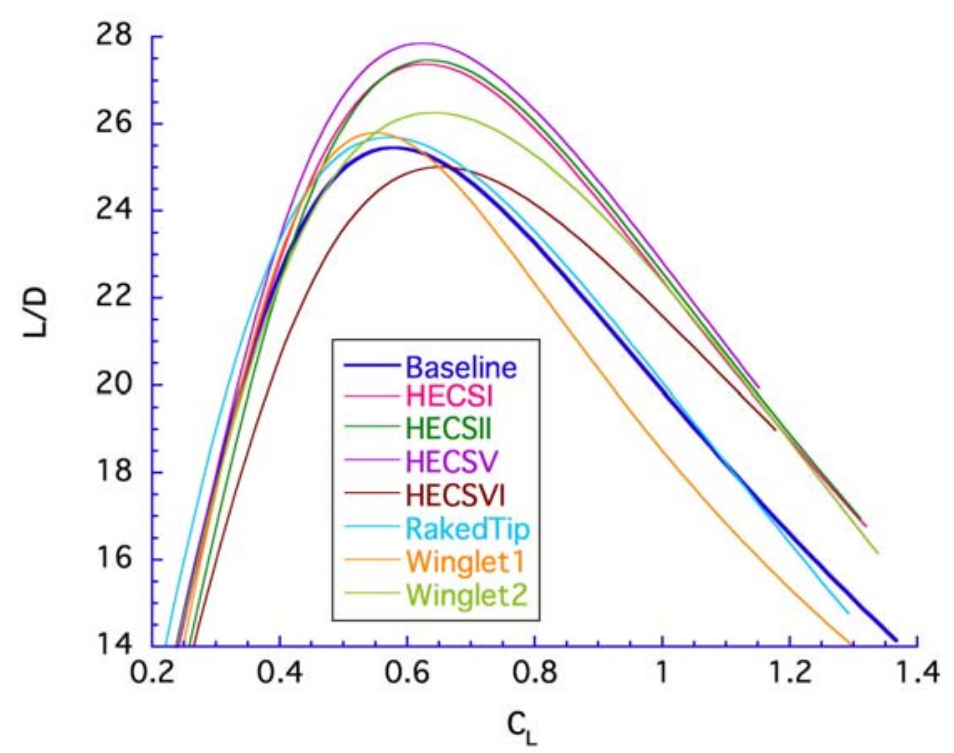

Figure 15. L/D performance for each configuration. $1^{\circ} \leq \alpha \leq 4^{\circ}$ where all of the configurations exhibited maximum liftto-drag performance. All of the lift curves are offset below the Baseline curve except for that of the Winglet1 configuration, however the lift curve slopes are remarkably similar. The lift trends are generally reflected in the drag data of Fig. 14a and $14 \mathrm{~b}$ in that a configuration with more lift has correspondingly more drag. The HECSV configuration demonstrates the lowest drag, whereas the Winglet1 configuration demonstrates the highest drag, which corresponds to its consistently higher lift for a given angle-of-attack.

A good performance rating for the tested wing configurations is the lift-todrag ratio, $\mathrm{L} / \mathrm{D}$. These values are plotted in Fig. 15 against lift coefficient, $\mathrm{C}_{\mathrm{L}}$. Table 2 shows the maximum $\mathrm{L} / \mathrm{D}$ values as well as the lift coefficient and angle-

of-attack where each maximum occurs. All configurations, except the HECSVI wing, performed better than the Baseline. The best performer was the HECSV wing with a maximum lift-to-drag ratio $\left(\mathrm{L} / \mathrm{D}_{\max }\right)$ of 27.85 . It is interesting to note that the configuration difference between the best performer, the HECSV wing, and the worst performer, the HECSVI wing, was a small amount of tip twist. Further comments about this will be made later in this section. Note also that the Baseline, Raked Tip, and Wingletl configurations reach a peak L/D value at lower lift coefficients than the other configurations. These configurations are less contoured than the others and the drag

Table 2. Maximum lift-to-drag values and percentage difference from Baseline value.

\begin{tabular}{lccccc}
\hline Wing model & $\mathbf{C}_{\mathbf{L} \text { L/D max }}$ & $\boldsymbol{\alpha}_{\mathbf{L} / \mathbf{D} \text { max }}$ & $\mathbf{L} / \mathbf{D}_{\max }$ & $\begin{array}{c}\text { Uncertainty } \\
\text { in L/D } \mathbf{m a x}\end{array}$ & $\begin{array}{c}\text { \% difference } \\
\text { from Baseline } \\
\text { in L/D } \mathbf{m a x}\end{array}$ \\
\hline Baseline & 0.574 & $1.77^{\circ}$ & 25.45 & \pm 0.7 & 0.0 \\
\hline HECSI & 0.630 & $2.96^{\circ}$ & 27.38 & \pm 0.8 & 7.6 \\
\hline HECSII & 0.638 & $2.67^{\circ}$ & 27.47 & \pm 0.8 & 9.9 \\
\hline HECSV & 0.618 & $2.93^{\circ}$ & 27.85 & \pm 0.9 & -1.8 \\
\hline HECSVI & 0.653 & $3.10^{\circ}$ & 25.01 & \pm 0.7 & 0.9 \\
\hline Raked Tip & 0.576 & $2.53^{\circ}$ & 25.69 & \pm 0.7 & 1.3 \\
\hline Winglet1 & 0.549 & $1.09^{\circ}$ & 25.79 & \pm 0.8 & 3.2 \\
\hline Winglet2 & 0.639 & $2.75^{\circ}$ & 26.26 & \pm 0.7 & \\
\hline \hline
\end{tabular}

polars in Fig. 16 show that in the range $0.4 \leq \mathrm{C}_{\mathrm{L}} \leq 0.9$, they exhibit a greater increase in drag for a given lift increment than do the other more contoured configurations.

Improvements in $\mathrm{L} / \mathrm{D}$ above the Baseline may be attributed to reductions in the induced drag component, $\mathrm{C}_{\mathrm{D} i}$, as the wetted areas were all greater than the Baseline. This may be expressed as an improvement in the effective aspect ratio and/or span load efficiency, $e$, a value difficult to determine from balance measurements. However, effective 
aspect ratio can be estimated from the out-of-plane extension of the wing, assuming $e$ stays the same, which, of course, is not necessarily the case.

In the following the induced drag reduction benefit due to an increase in effective aspect ratio is estimated through the hypothetical use of out-of-plane tip extensions. Consider two wing configurations: one with geometric and aerodynamic characteristics of the Baseline and the other a configuration similar to the Baseline in all respects except vertical tip extensions are included with length equal to that of the HECS configurations (4.727 in.). The change in $\mathrm{C}_{\mathrm{Di}}$ may be written as

$$
\Delta C_{D i}=C_{D i_{2}}-C_{D i_{1}}=\frac{C_{L}^{2} S}{\pi e}\left(\frac{1}{b_{2}^{2}}-\frac{1}{b_{1}^{2}}\right) .
$$

For the sake of argument, and the most conservative estimate, the efficiency rating for both configurations will be set to $e=1$, and an equivalent $\mathrm{C}_{\mathrm{L}}$ and planform area (those of the Baseline) will be used. The Baseline configuration has a wingspan of $b_{1}=28$ inches, a $C_{L}$ of 0.574 at $L / D_{\max }$, and a planform area of $S=112 \mathrm{in}^{2}$. From Ref. 7, a span benefit of $45 \%$ of the tip extension length can be estimated, roughly half the length of the extension. Therefore, for the theoretical configuration of the Baseline with tip extensions, $b_{2}=2 *(14+4.727 * 0.45)=32.25$ in. resulting in

$$
\Delta C_{D i}=C_{D i_{2}}-C_{D i_{1}}=\frac{0.574^{2}(112)}{\pi e}\left(\frac{1}{32.25^{2}}-\frac{1}{28^{2}}\right)=-0.0037
$$

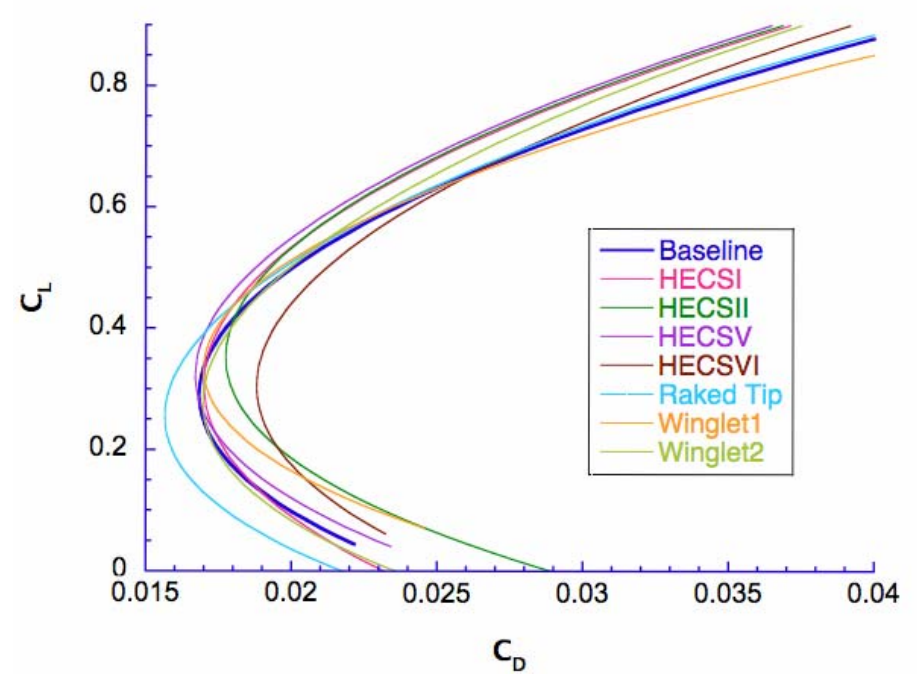

Figure 16. Drag polars of the tested wing configurations

which indicates a reduction of $37 \mathrm{drag}$ counts. At the given condition, the Baseline wing exhibits an $\mathrm{L} / \mathrm{D}$ of 25.45 and a drag coefficient of $\mathrm{C}_{\mathrm{D}}=0.0225$. A reduction in $C_{D}$ of 0.0037 would yield an ideal improvement in $\mathrm{L} / \mathrm{D}$ to 30.46. Of course this is tempered by the increase in profile drag, but indicates the performance of the HECS wing configurations falls within what is possible.

It should reiterated at this point that an error in winglet height design resulted in model tip extensions of $\mathrm{z}=$ 4.227 in. instead of the desired $\mathrm{z}=$ 4.727 in., a reduction of 0.5 in. less than that of the HECS wing configurations. This unfortunate error can be argued to compromise the capability of the winglet configurations. An estimate of the difference in $\mathrm{C}_{\mathrm{Di}}$ by an additional 0.5 inch tip extension can be performed using the above argument with $\mathrm{C}_{\mathrm{L}}$ values of 0.549 and 0.639 for Winglet1 and Winglet 2 configurations, respectively. The results show a 3 count drag reduction for Winglet1, and a 4 count drag reduction for Winglet 2. This translates to an improvement in $\mathrm{L} / \mathrm{D}_{\max }$ for Winglet1 from 25.79 to 26.14 and an improvement in $\mathrm{L} / \mathrm{D}_{\max }$ for Winglet2 from 26.26 to 26.70 . These calculations do not, of course, account for the profile drag penalty.

The profile drag characteristics of each wing configuration may be investigated by considering minimum drag values, $\mathrm{C}_{\mathrm{D}(\min )}$. Figure 16 shows the drag polar for each configuration and $\mathrm{C}_{\mathrm{D}(\min )}$ values are determined by locating the point on each curve where $d C_{D} / d C_{L}$ is zero. For the speed regime and model characteristics under consideration, profile drag includes both skin friction and form drag, where form drag is drag due to viscous separation. If form drag is small, i.e., there is minimal flow separation, there should be a good correlation between the values of $\mathrm{C}_{\mathrm{D}(\min )}$ and wetted surface area. Table 3 shows values of $\mathrm{C}_{\mathrm{D}(\min )}$ for each wing configuration along with percentage differences compared to the Baseline wing. The surface area of each wing was calculated with the CAD 
software used to build the wings and is also shown in the table. The $\mathrm{C}_{\mathrm{D}(\min )}$ values have an uncertainty of approximately \pm 0.0005 . All surface area values are in inches squared and represent the surface area of a single wing.

As can be seen in Table 3, the Raked Tip configuration has the lowest value of $\mathrm{C}_{\mathrm{D}(\min )}$ and the HECSVI configuration has the highest value. Both values fall outside the error band. For all other configurations, values of

Table 3. Minimum drag coefficient from polar, Fig. 16

\begin{tabular}{lcrrr}
\hline \hline Wing model & $\mathbf{C}_{\mathbf{D}(\mathbf{m i n})}$ & $\begin{array}{c}\text { Percent } \\
\text { difference } \\
\left(\mathbf{C}_{\mathbf{D}(\mathbf{m i n})}\right)\end{array}$ & $\begin{array}{c}\text { Wing surface } \\
\text { area, in }\end{array}$ & $\begin{array}{c}\text { Percent } \\
\text { difference } \\
\text { (Surface area) }\end{array}$ \\
\hline Baseline & 0.0168 & 0.0 & 114.206 & 0.0 \\
\hline HECSI & 0.0170 & 1.2 & 117.911 & 3.2 \\
\hline HECSII & 0.0177 & 5.4 & 117.911 & 3.2 \\
\hline HECSV & 0.0167 & -0.6 & 126.690 & 10.9 \\
\hline HECSVI & 0.0188 & 11.9 & 126.690 & 0.3 \\
\hline Raked Tip & 0.0157 & -6.5 & 114.596 & 7.8 \\
\hline Winglet1 & 0.0170 & 1.2 & 123.071 & 6.2 \\
\hline Winglet2 & 0.0170 & 1.2 & 121.281 & \\
\hline \hline
\end{tabular}

$C_{D(\min )}$ are within a few drag counts of each other and fall within the error band. A comparison of $C_{D(\min )}$ values with wetted surface area values shows there is no clear correlation between the two. From this it can be inferred that drag due to flow separation plays an important roll in the current study.

It is also interesting to note differences in $\mathrm{C}_{\mathrm{D}(\min )}$ for configurations with the same wetted surface area. For the current study there are two such pairs. The HECSI and HECSII configurations both have equal wetted surface areas as do the HECSV, HECSVI configurations. Recall that the only difference between the HECSI and HECSII configurations is minor tip twist in the latter. From Table 3, the HECSII wing has a larger $\mathrm{C}_{\mathrm{D}(\min )}$ value than that for the HECSI wing. While the values are within the error band, a trend can certainly be inferred indicating tip twist results in more flow separation. However, these two configurations exhibit comparable performance as shown in the

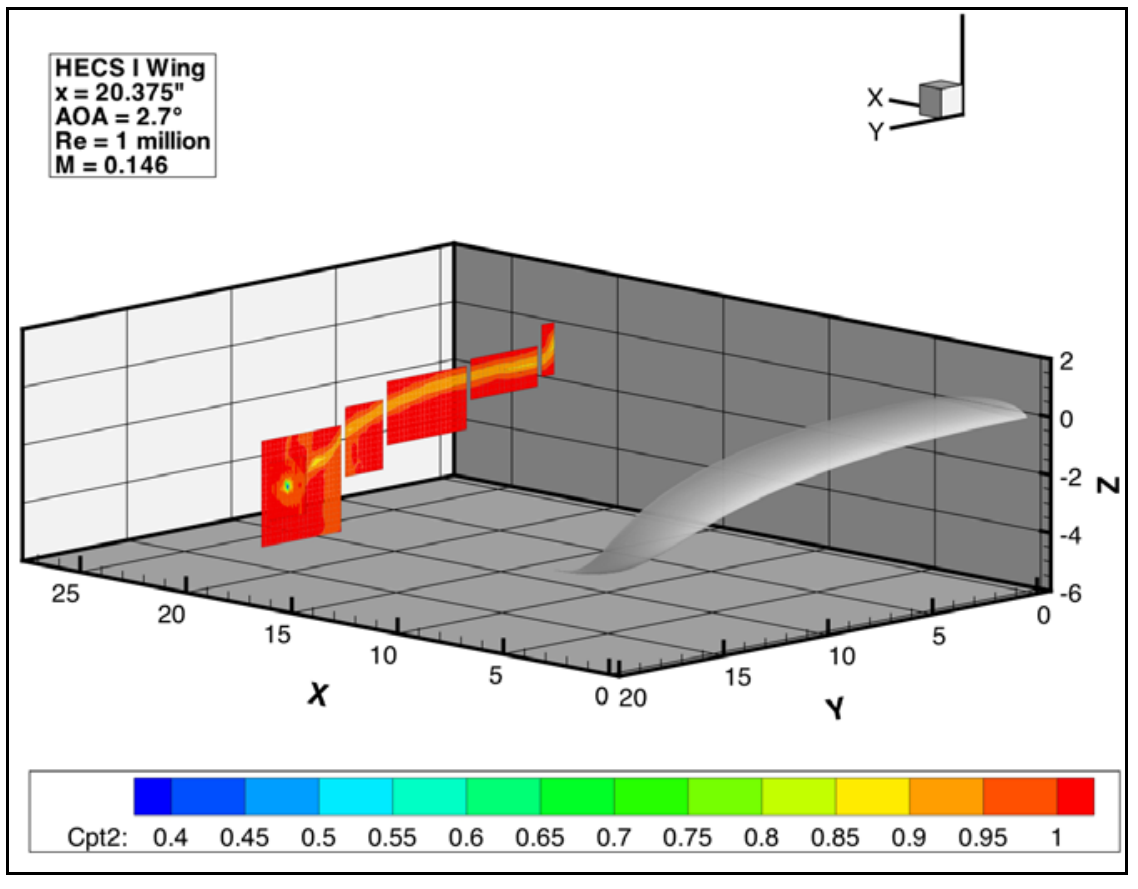

Figure 17. Seven hole probe wake pressure survey location aft of HECSI lift-to-drag plot of Fig. 15, the HECSII wing providing a slightly better performance. While we can only consider the trends within the error band, it may be hypothesized that a trade off exists between higher form drag and lower induced drag. Where the crossover point exists is topic for further investigation.

Consider now the HECSV and HECSVI configurations, which also have the same wetted surface area. As noted previously, the HECSV wing performed somewhat erratically, particularly in the drag bucket where measurements at times did not repeat well. From the flow field studies, presented in the next section, it appears that at least two different flow states existed and it is suspected that flow separation was the basis 
of transition from one state to another. This theory is somewhat supported by the results of Table 3 which show a much higher $\mathrm{C}_{\mathrm{D}(\min )}$ value for the HECSVI wing than the HECSV suggesting that there is significant flow separation on the HECSVI configuration. The minor tip twist in the HECSVI wing may have resulted in a more fully separated flow state, one to which the HECSV wing transitions to and from.

\section{B. Flow Field Data}

In an effort to understand the wake flow physics behind some of the configurations, seven hole probe wake surveys were conducted at a station $x=20.375$ inches downstream of the wing root leading edge. The configurations for which wake data were acquired included the Baseline, HECSI, HECSII, HECSV, HECSVI, and Winglet2. Figure 17 illustrates an example grid distribution taken at $x=20.375$ inches downstream of the HECSI configuration. Coarse and fine grids were acquired at this location and resampled to a single grid using weighted averaging techniques as noted previously. Fine grid data were also acquired in a plane at $x=11.25$ inch for the HECSII configuration to track the tip vortex. HECSVI wake data were acquired in an effort to determine why this configuration performed so poorly but the data provided no insight and is therefore not presented. Flow field parameters were non-dimensionalized in the manner of $\mathrm{C}_{\mathrm{pt}}, \mathrm{u} / \mathrm{U}_{\infty}$, and $\Omega \mathrm{c} / \mathrm{U}_{\infty}$.

Figure 18 illustrates the total pressure distributions as obtained in the wake for all configurations of interest. The most noticeable difference is in the location of the tip vortex, highlighted by the large reduction in $\mathrm{C}_{\mathrm{pt}}$. While the Baseline wing core location is inboard at $y /(\mathrm{b} / 2)=0.95$ and above the $z=0$ datum at $z /(\mathrm{b} / 2)=0.06$, the HECSV wing tip vortex is located 6 percent outboard of the span at a $y /(\mathrm{b} / 2)=1.06$ and below the datum at $z /(\mathrm{b} / 2)=-0.27$. The impact of this distance on the downwash of the wing, in particular the inboard regions, could substantially affect the induced drag. Note in this figure the two stations of data for the HECSII configuration. At station 1 upstream, the $y-z$ location of the vortex is very near the model tip whereas further downstream the vortex has translated both in the positive $y$ - and $z$-directions. It is also interesting to note that the wake of the winglet does not display a vortex core as intense as any of the other configurations.

The corresponding normalized axial velocity distributions are shown in Fig. 19. Most of the configurations indicate a vortex core velocity at or below free stream with an elevated region of velocity outboard of the core. This is not necessarily a physical phenomenon and may be a manifestation due to the presence of the probe. Most notable is the contrast in the data taken on the HECSV wing. The coarse grid data is completely unlike the fine grid, the latter more similar to the other flows. It is proposed that these two different wake flows reflect two states of flow alluded to earlier for this configuration. It is unknown whether the coarse grid or the fine grid case produced the best L/D performance, as the model was not on the balance when the wake data were acquired. No explanation for the very high axial flow exhibited in the coarse grid case was apparent to the investigators. The winglet did not demonstrate the level of axial flow deficit as was seen in the other wings, excluding the coarse grid HECSV.

The cross-plane velocity field was differentiated to determine the axial vorticity field. It is shown in Fig. 20 normalized with mean-aerodynamic-chord and freestream velocity. Not much difference is seen in the fine to coarse grid comparison of the HECSV wing, except for perhaps the effects of increased grid resolution. This would indicate the cross flow velocities for the two different flow states do not vary as much as the axial velocity component. Indeed, contour plots of normalized $\mathrm{v}$ and $\mathrm{w}$ velocities (not shown) indicate only a minimal change in the $\mathrm{v}$ component with no discernable difference in the w-component. Figure 15 shows the HECSI and HECSII wings display quite similar L/D performance levels. Figure 20 indicates the HECSII vortex is weaker and slightly offset from the position of HECSI vortex in the negative $\mathrm{z}$ direction. A closer look at Fig. 15 illustrates the HECSII has a slightly better performance, but the effect on the span load is difficult to ascertain.

Although this did not fall under the focus of this investigation, the implication of the lower vorticity levels in the winglet wake is interesting to contemplate. If this is indeed the case, the presence of a winglet, or at least this winglet, could be used to mitigate the formation of a strong tip vortex and decrease the dangers associated with aircraft passing through the wake of another plane, in addition to providing an induced drag benefit. 


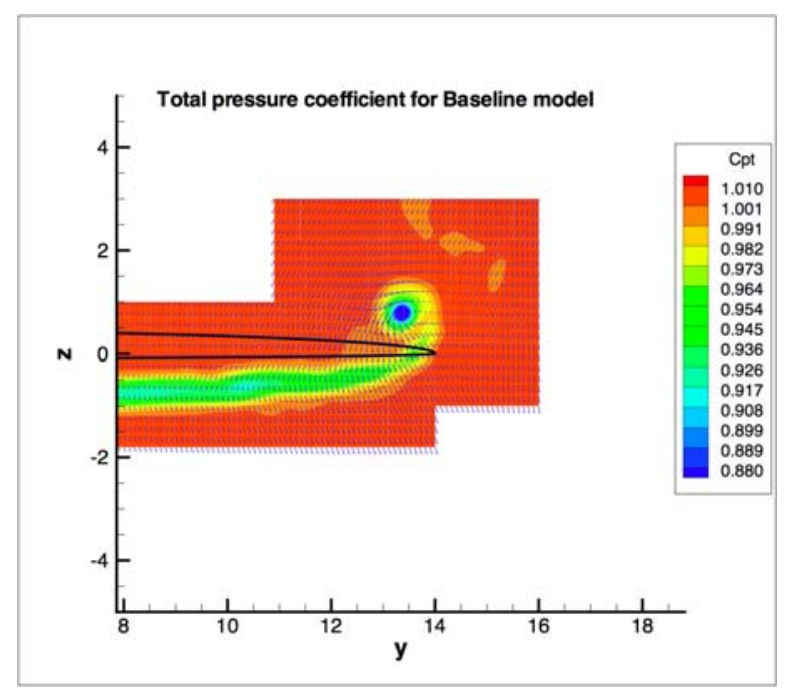

a) Baseline

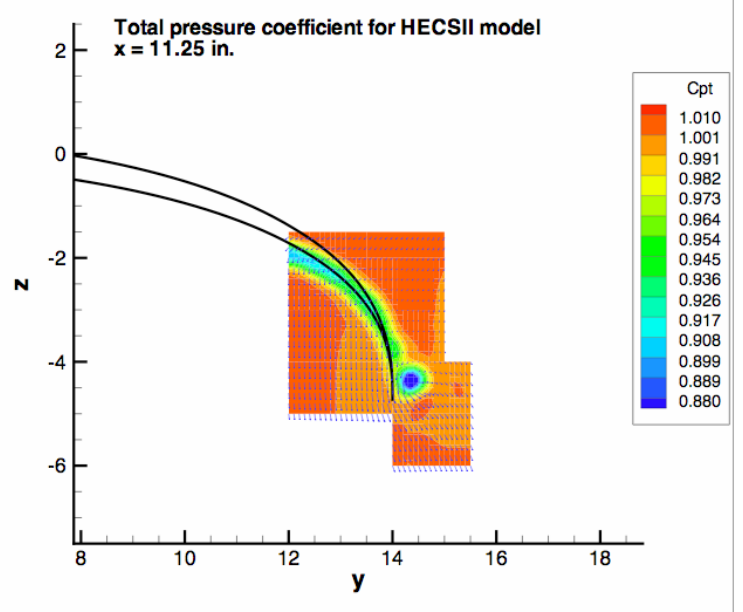

c) HECSII, station 1 at $x=11.25$ in.

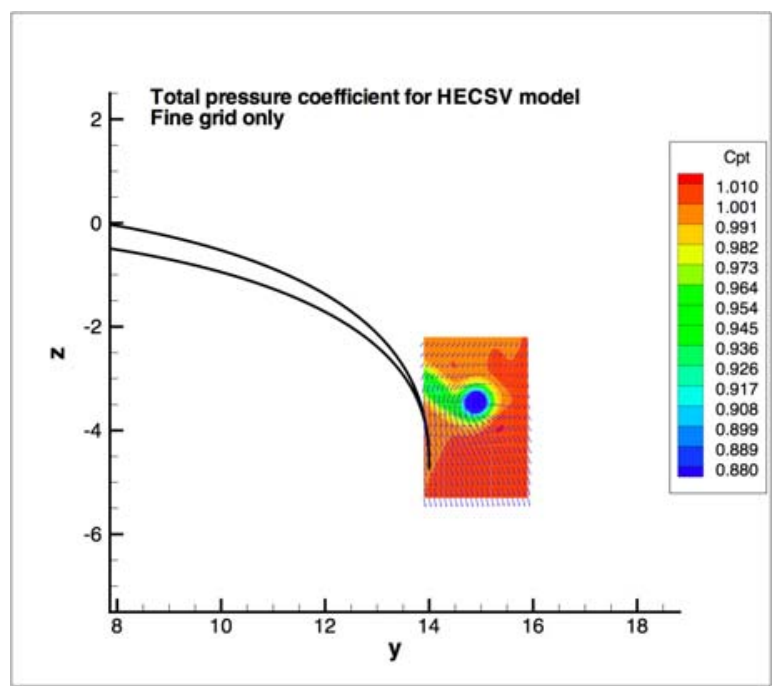

e) HECSV

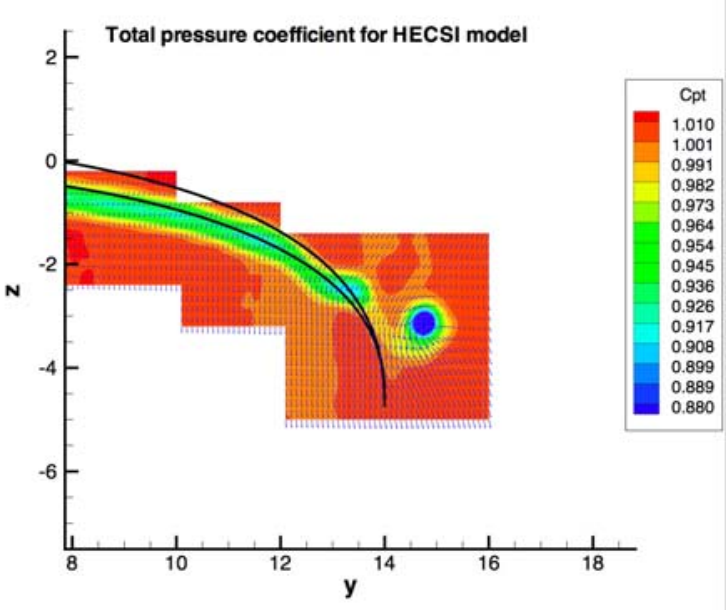

b) HECSI

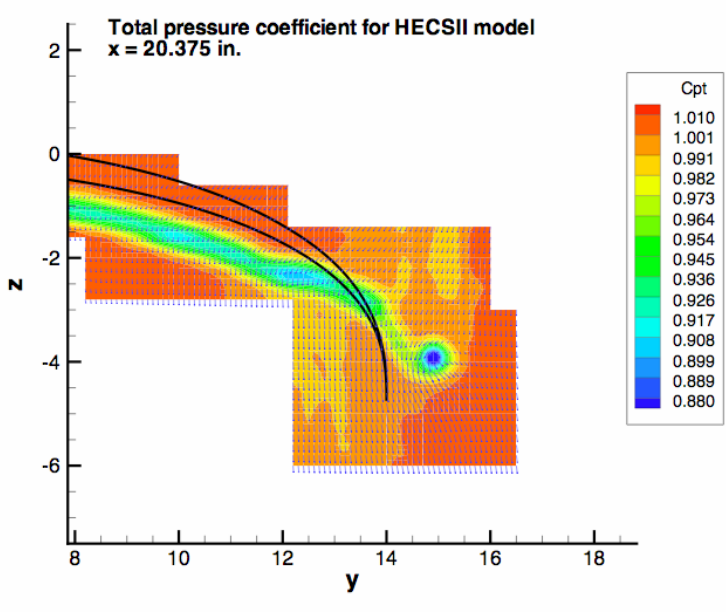

d) HECSII, station 2 at $x=20.375$ in.

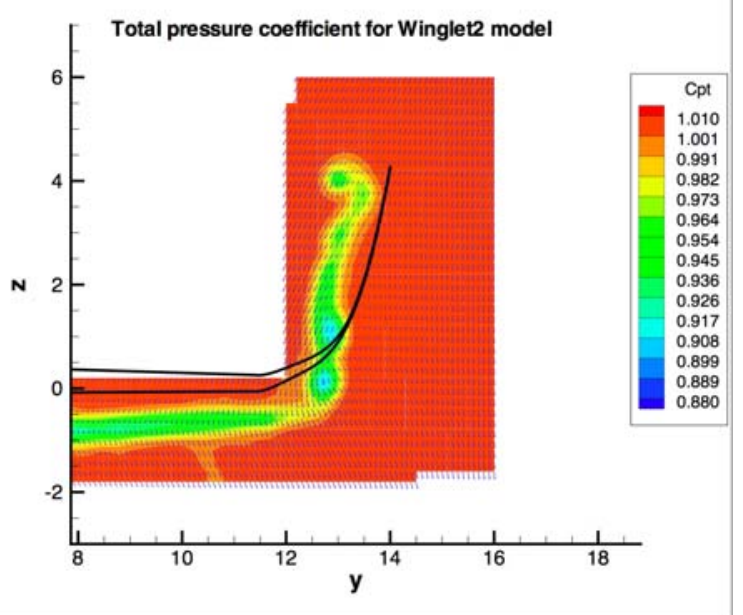

f) Winglet2

Figure 18. Seven hole probe total pressure results 


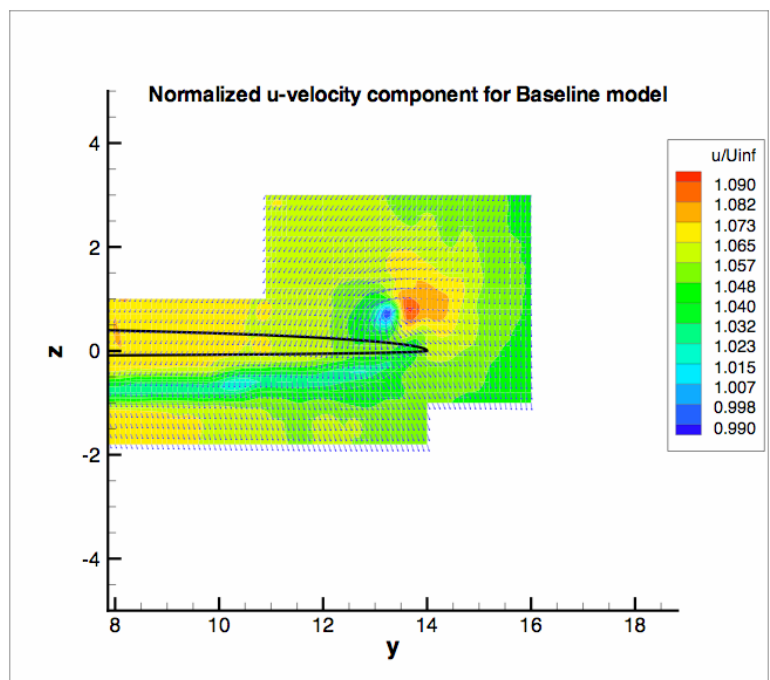

a) Baseline

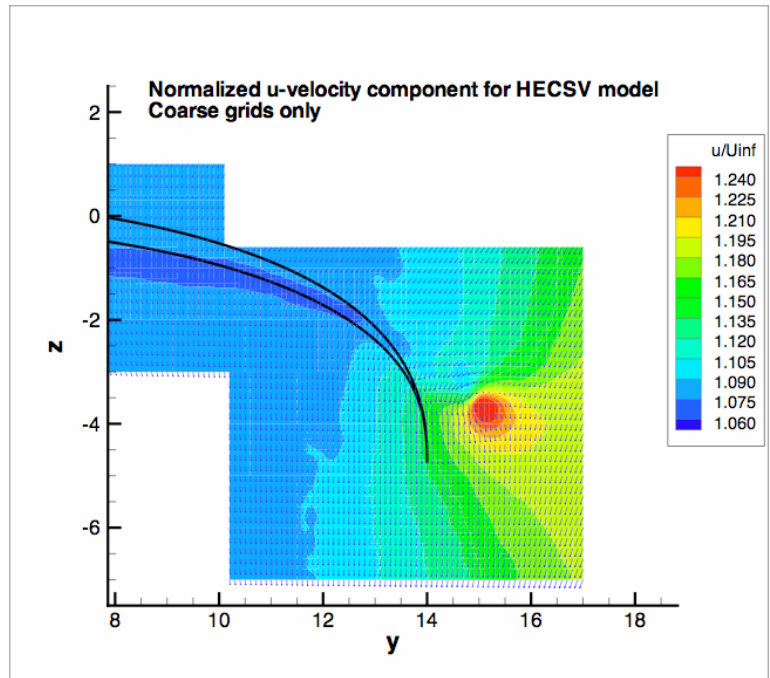

c) HECSV, coarse grids

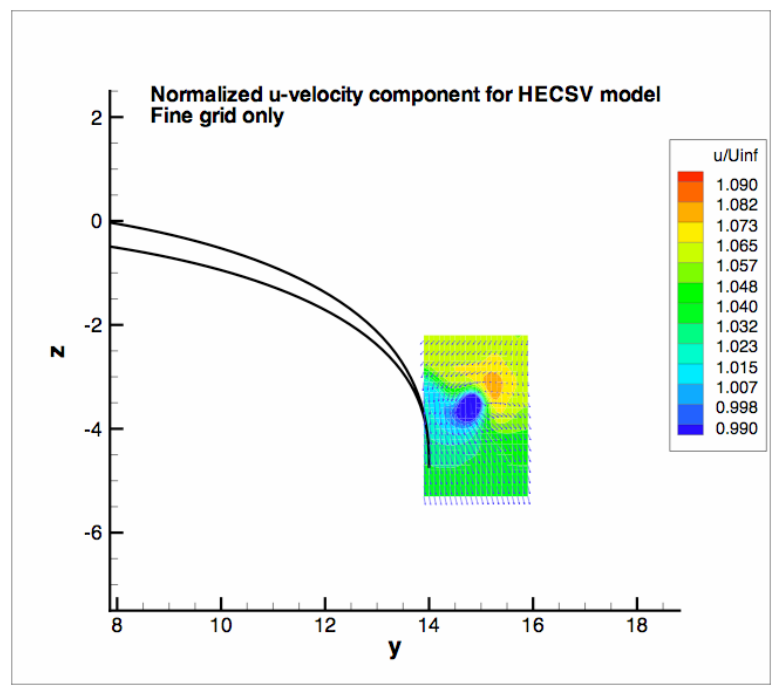

e) HECSV, fine grid

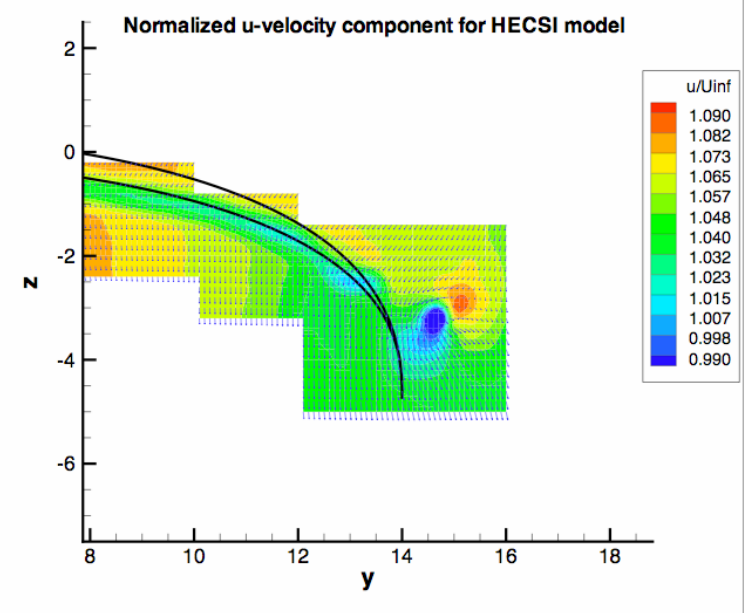

b) HECSI

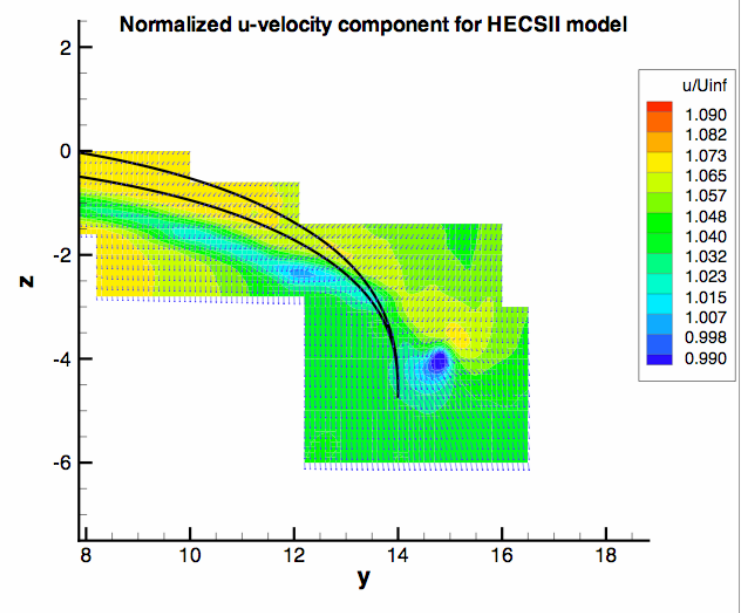

d) HECSII

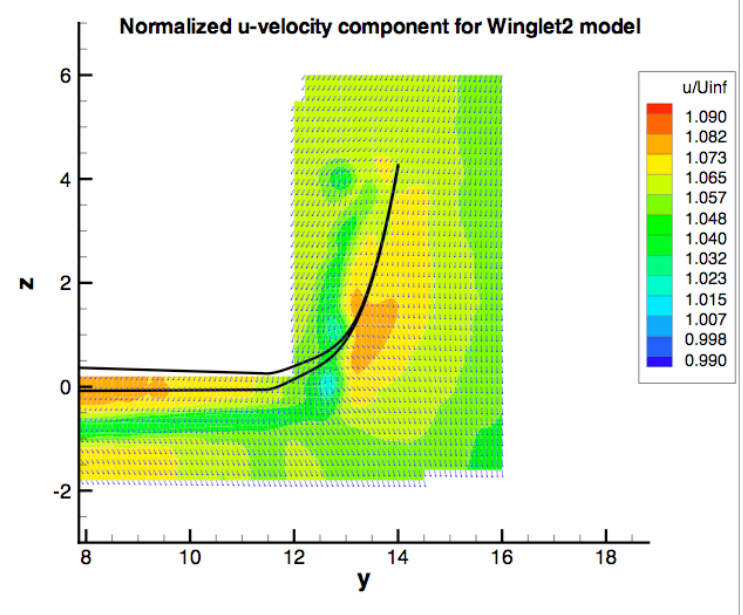

f) Winglet2

Figure 19. Seven hole probe axial velocity results 


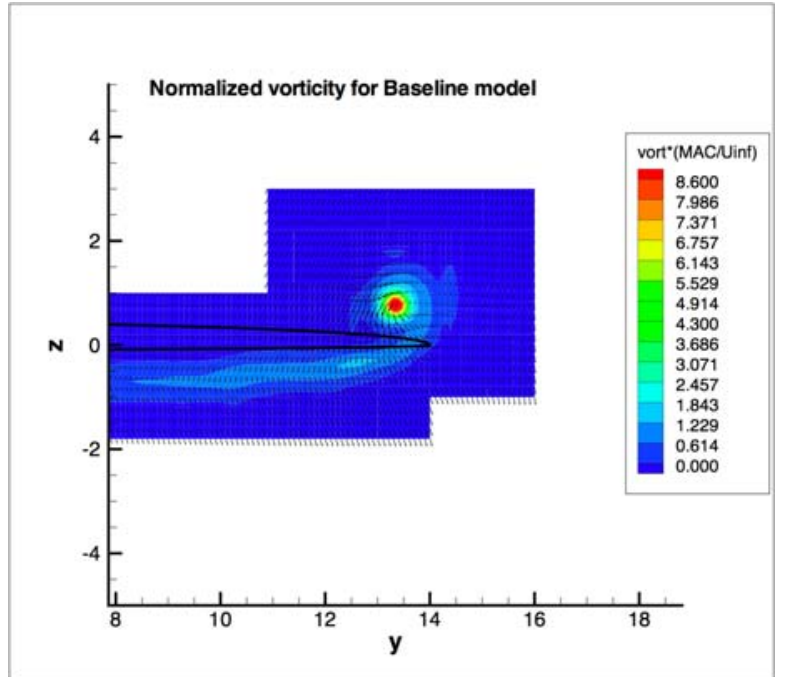

a) Baseline

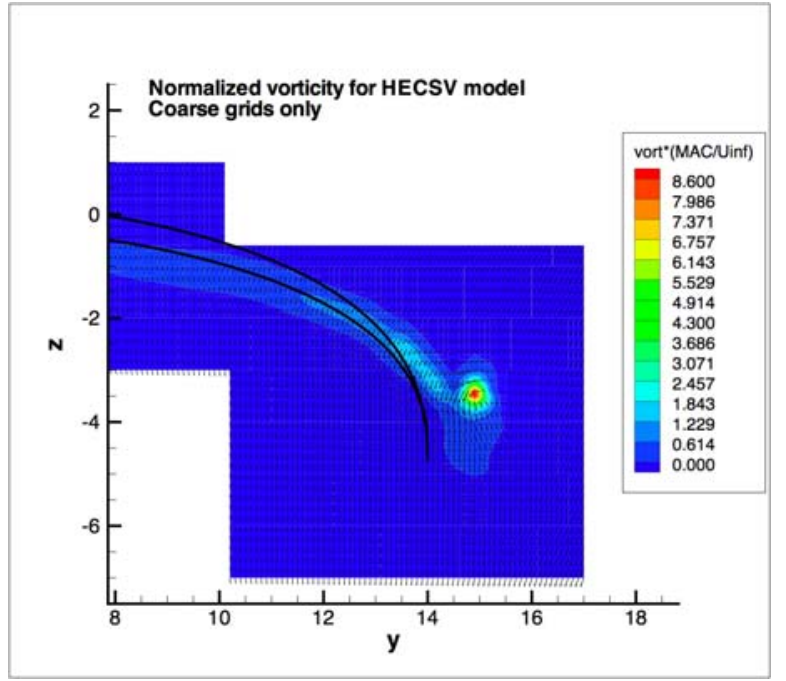

c) HECS, coarse grid

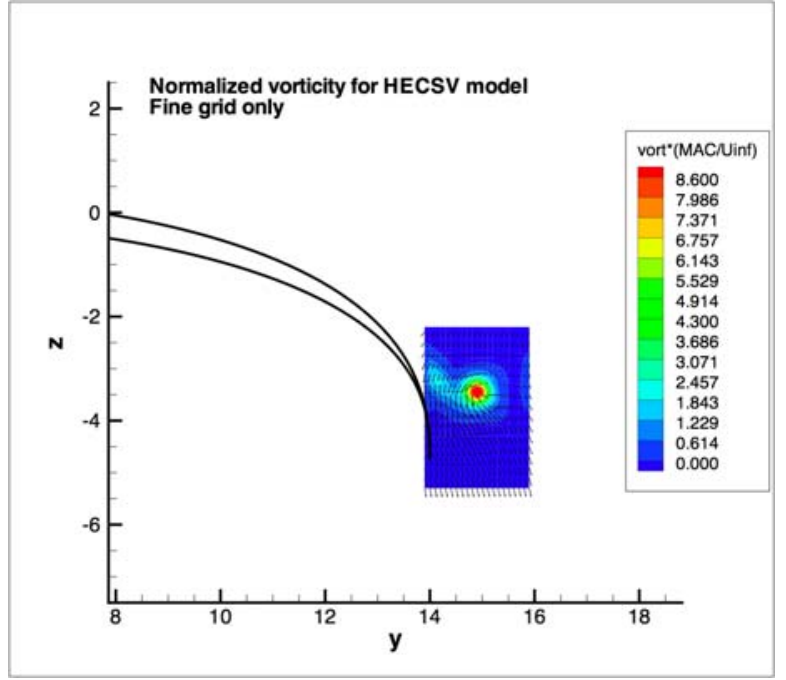

e) HECSV, fine grid

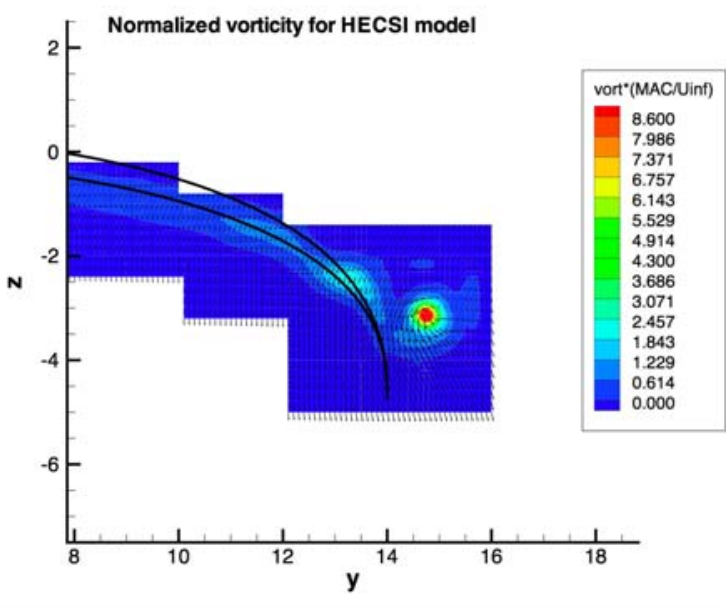

b) HECSI

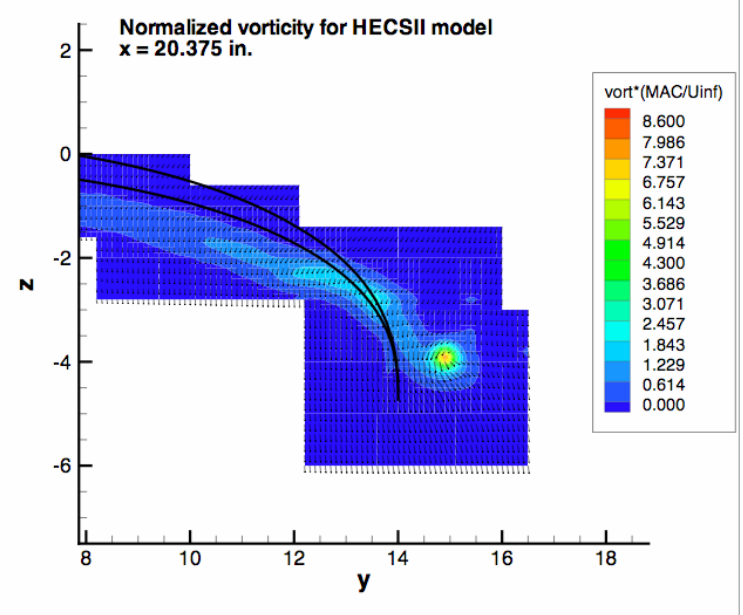

d) HECSII, station 2 at $x=20.375$ in.

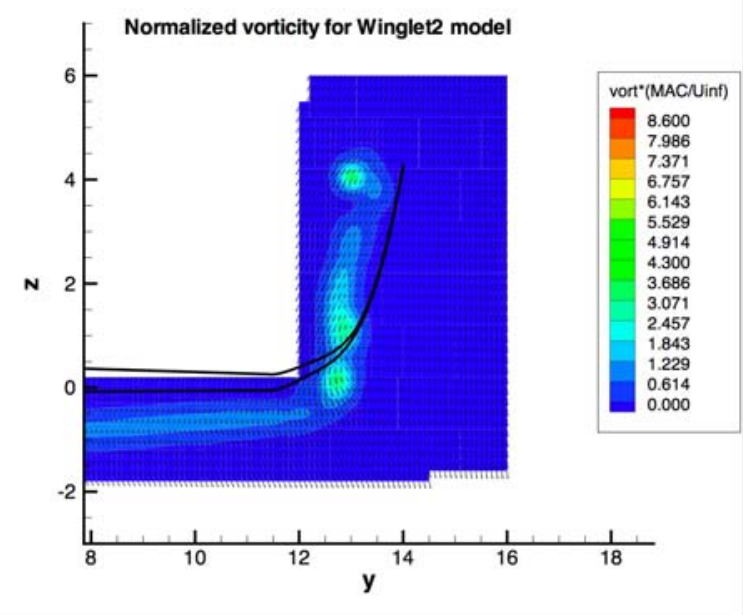

f) Winglet2

Figure 20. Seven hole probe vorticity wake results 


\section{Summary and Conclusions}

An experimental study was conducted to determine the aerodynamic performance and wake characteristics of hyper-elliptic cambered span (HECS) wings and configurations of more conventional design. Models tested included the HECSI and HECSII configurations, which were unswept. The HECSII configuration was identical to the HECSI but included a minor tip twist, a modification similar to the toe angle on a winglet. The HECSV and HECSVI configurations were swept by offsetting each wing section in the streamwise direction according to a specified criterion. These two configurations were identical except for a minor tip twist in the HECSVI configuration similar to HECSII. More conventional configurations tested included a Baseline that was elliptic in planform with a straight trailing edge, two winglet configurations, and a raked tip. All configurations had equal planform area and aspect ratio to provide a good comparison. Models were sting mounted to a strain gauge balance and run through an angle-of-attack range to acquire force and moment data. Wake surveys were conducted behind several of the configurations using a seven-hole probe to identify flow features.

In a lift-to-drag comparison, all configurations outperformed the Baseline (elliptic planform) except the HECSVI configuration. The HECSV demonstrated the best performance but both balance and wake data suggest this configuration develops a bi-stable flow field resulting in either superior or inferior performance. The HECSI and HECSII configurations were the next best performers with the HECSII performing slightly better. Of the two winglet configurations, Winglet2 performed the best, maintaining a lift-to-drag improvement over a broad range of the lift coefficient. Winglet1 outperformed the Baseline at the lower $\mathrm{C}_{\mathrm{L}}$ values but dropped precipitously below Baseline values as $C_{L}$ increased. The raked tip configuration showed only slight improvement over the Baseline but demonstrated the lowest minimum drag value.

Flow field data in a $y$-z plane located 20.375 inches downstream of the wing root leading edge highlighted significant differences in the strength and core location of the wing tip vortex. The core location on the Baseline was above the wing and inboard of the tip as would be expected. The vortex core on the HECSI and HECSII wings was displaced outboard of the wing and significantly below the wing root. Comparing results from a survey conducted further upstream it was noted that the HECSII tip vortex migrates outboard from its origin. Qualitatively the vortex strength for the HECSI wing was similar to that of the Baseline but the vortex strength of the HECSII wing was diminished. Vorticity at the tip of the Winglet2 configuration was diffuse and lower in magnitude than the other configurations tested suggesting it may be useful for vortex wake hazard mitigation as well

Although not yet optimized from a span load point of view, the HECS geometry, which is reflective of configurations found in the avian community, may represent a class of out-of-plane configurations that could exceed the performance of conventional winglets. It is proposed that observed tip vortex displacement reduces the induced drag for an overall net improvement in the lift-to-drag ratio for HECS configurations. Further improvements may be obtained by twisting the tip of the HECS wing, which tends to diminish the tip vortex strength.

\section{Acknowledgments}

The support of NASA Langley Research Center Grant NAS1-02117 through the National Institute of Aerospace is greatly appreciated.

\section{References}

${ }^{1}$ Munk, M. M., “The Minimum Induced Drag of Aerofoils,” NACA Report No. 121, 1923.

${ }^{2}$ Cone, C. D., "The Theory of Induced Lift and Minimum Induced Drag of Non-planar Lifting Systems," NACA Technical Report R-139, 1962.

${ }^{3}$ Whitcomb, R. T., "A Design Approach and Selected Wind Tunnel Results at High Subsonic Speeds for Wing-Tip mounted Winglet", NASA TN-D-8260, 1976.

${ }^{4}$ Maughmer, M. D., "The Design of Winglets for High Performance Sailplanes", AIAA 2001-2406, December 2000

${ }^{5}$ Jarvis, J., and Selberg, B. "Investigation of Aerodynamic Improvements Using Wing Tip Sails", 37th AIAA Aerospace Sciences Meeting, AIAA 99-0530 January 11-14, 1999 Reno, Nevada.

${ }^{6}$ Jones, R., and Lasinski, T. "Minimum Induced Drag of Wings with Winglets" NASA TM-81230, September 1980.

${ }^{7}$ Kroo, I. "Design and Analysis of Optimally Loaded Systems", AIAA 84-2507 October 1984.

${ }^{8}$ Norton, F.H., "An Investigation on the Effect of Raked Wing Tips", NACA-TN-69, Nov.,1921.

${ }^{9}$ Vijgen, P.M.H.W, Van Dam, C.P., and B. Holmes. "Sheared Wing-Tip Aerodynamics: Wind Tunnel and Computational Investigation." Journal of Aircraft, Vol. 26, No. 3. March 1989.

${ }^{10}$ VanDam, C. P., "Induced-Drag Characteristics of Crescent-Moon-Shaped Wings", Journal of Aircraft, Vol.24, No.2, Feb 1987.

${ }^{11}$ VanDam, C. P., Vijgen, P.M. H. W., Holmes, B. J., "Wind Tunnel Investigation on the Effect of Crescent Planform Shape on Drag, AIAA 90-0300 
${ }^{12}$ Smith, S.C. and Kroo, I.M., “A Closer Look at the Induced Drag of Crescent - Shaped Wings”, AIAA 90-3063, $8^{\text {th }}$ Applied Aerodynamics Conference, Portland, OR, August 20-22, 1990

${ }^{13}$ Henderson, W. P., and Holmes, B. J., "Induced Drag - Historical Perspective,” SAE Paper 892341, Sept. 1989.

${ }^{14}$ Rokhsaz, K., “A brief Survey of Wingtip Devices for Drag Reduction,” SAE Paper 932574, Sept. 1993.

${ }^{15}$ Lazos, B. S., "Biologically Inspired Fixed-Wing Configuration Studies," Journal of Aircraft, Vol. 42, No. 5, Sept-Oct, 2005.

${ }^{16}$ Sellers, W. L., and Kjelgaard, S. O., "The Basic Aerodynamics Research Tunnel - A Facility Dedicated to Code Validation," AIAA Paper 88-1997, 1988

${ }^{17}$ Selig, M. S., Guglielmo, J. J., Broeren, A. P., and Giguere, P., "Summary of Low-Speed Airfoil Data," Vol. 1, http://www.nasg.com/afdb/show-polar-e.phtml.

${ }^{18}$ Smith, S. C., "A Computational and Experimental Study of Nonlinear Aspects of Induced Drag," NASA Technical Paper No. 3598, Feb. 1996

${ }^{19}$ Melin.T, Tornado, "A vortex lattice MATLAB implementation for Linear Aerodynamic Wing applications", Masters thesis, Royal Institute of Technology (KTH), Sweden, December 2000

${ }^{20}$ Moran, J "Computational Fluid Dynamics", Weily\&Sons, 1984

${ }^{21}$ Visser, K.D. "Wing Tip Research", National Institute of Aerospace Final Report NASA \#NNL04AC97T, January 2006.

${ }^{22}$ Barlow, J. B., Rae, W. H., and Pope, A., "Forces and Moments From Balance Measurements," Low-Speed Wind Tunnel Testing, $3^{\text {nd }}$ Edition, Wiley \& Sons Inc., 1999, pp. 265-271. 